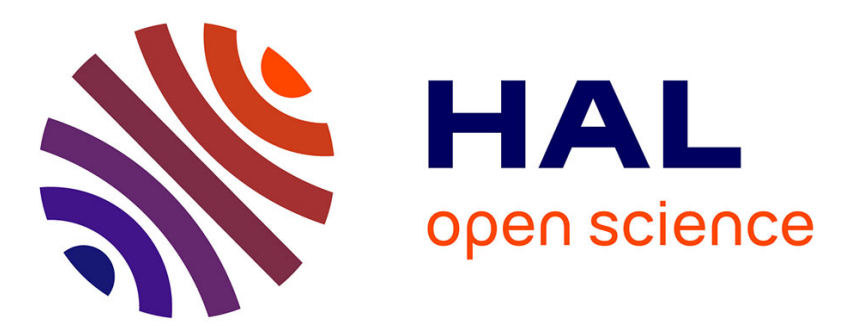

\title{
An anisotropic viscoelastic fibre-matrix model at finite strains: Continuum formulation and computational aspects
}

Boumediene Nedjar

\section{- To cite this version:}

Boumediene Nedjar. An anisotropic viscoelastic fibre-matrix model at finite strains: Continuum formulation and computational aspects. Computer Methods in Applied Mechanics and Engineering, 2007, 196 (9-12), pp.1745-1756. 10.1016/j.cma.2006.09.009 . hal-00141142

HAL Id: hal-00141142

https://hal.science/hal-00141142

Submitted on 11 Apr 2007

HAL is a multi-disciplinary open access archive for the deposit and dissemination of scientific research documents, whether they are published or not. The documents may come from teaching and research institutions in France or abroad, or from public or private research centers.
L'archive ouverte pluridisciplinaire HAL, est destinée au dépôt et à la diffusion de documents scientifiques de niveau recherche, publiés ou non, émanant des établissements d'enseignement et de recherche français ou étrangers, des laboratoires publics ou privés. 


\title{
An anisotropic viscoelastic fibre-matrix model at finite strains: Continuum formulation and computational aspects
}

\author{
B. Nedjar ${ }^{1}$ \\ Laboratoire d'Analyse des Matériaux et Identification \\ Ecole Nationale des Ponts et Chaussées \\ 6 et 8, avenue Blaise Pascal, 77455 Marne la Vallée Cedex 2, France
}

\begin{abstract}
This paper presents a fully three-dimensional constitutive model for anisotropic viscoelasticity suitable for the macroscopic description of fibre reinforced composites that experience finite strains. An essential feature of the model is that the matrix and the fibres are treated separately allowing then as many bundles of fibres as desired. Moreover, the relaxation and/or creep response is based on the multiplicative viscoelastic split of the deformation gradient combined with the assumption of viscoelastic potentials for each compound. Here the composite is thought to be the superposition of an isotropic matrix material and further one-dimensional continua, each of them representing one family of fibres. The deformation gradient and its multiplicative decomposition apply to all the continua linking them implicitly. The global anisotropic response is obtained by an assembly of all the contributions. Constitutive models for orthotropic and transversely isotropic materials are included as special cases. It is shown how the continuum thermodynamics is crucial in setting the correct forms for the constitutive and evolution equations. For the algorithmic design within the context of the finite element method, the numerical effort is of the order of that devoted for isotropic computations. In fact, only a single scalarvalued resolution procedure is added for each fibre bundle. The algorithmic tangent moduli are derived for each compound and their assembly leads to consistent viscoelastic tangent modulus which is suitable for a quadratic rate of convergence when the Newton-Raphson iterative scheme is employed. The numerical efficiency of the model is illustrated through a set of representative simulations.
\end{abstract}

Key words: Anisotropic viscoelasticity, finite strains, fibre-matrix composite materials, multiplicative split of the deformation gradient, continuum thermodynamics.

1 E-mail: nedjar@lami.enpc.fr 


\section{Introduction}

Fibre-reinforced materials belong to a very important class of materials which are frequently employed in a wide variety of industrial applications. Typically, these materials consist of a fabric structure where the fibres are continuously arranged in a matrix material and, at the macroscopic level, these composites exhibit strong directional dependencies. The vehicle tyres furnishes a typical example of technological application of such man-made composites. On the other hand, focusing on biomechanical problems, a large number of elements of living organisms, such as all types of soft biological tissues, show very distinct anisotropic mechanical properties. Typically, these soft tissues are for example composed by many layers, each of them consists of many families of collagenous fibres with different orientations continuously embedded in a non-collagenous matrix material. Their study is nowadays of growing interest.

In many cases, these anisotropic materials behave in a viscoelastic manner in the finite strains range. The dissipative phenomena can either be exhibited by the fibres and/or by the matrix itself. In most cases, it is essential to consider the viscous effects within a phenomenological modelling framework. Recently, a considerable effort has been devoted into the extension of isotropic finite strain inelastic models to the anisotropic case from the theoretical and the numerical points of view. We refer for example to the anisotropic elastoplastic formulations proposed by Spencer (2001) [32], Reese (2003) [20], Sansour and Bocko (2003) [23], and more recently by Klinkel, Sansour and Wagner (2005) [11]. And for anisotropic viscoelasticity, we refer for example to the recent formulation by Holzapfel and Gasser (2001) in [8], see also [9]. In this latter, the authors have pursued Simo's constitutive framework [27] where the overstresses of each compound of the composite materials are chosen as internal variables which, in turn, are governed by linear differential equations in a form similar to that employed in linear viscoelasticity. In all these formulations, the anisotropic behaviour is, among others, captured by a particular choice of the free energy function formulated in terms of the so-called integrity basis as proposed by Spencer (1984) [31], and widely employed for purely elastic materials, see for example $[1,22,34]$ to mention few.

The goal of the present paper is an alternative formulation of the viscoelastic response of fibre-reinforced materials at finite strains. Two features characterize the present approach. On the one hand, it extends the hypothesis of the multiplicative split of the deformation gradient into an elastically relaxing part and a viscous part to all the compounds of the composites. Note that this multiplicative decomposition, originally proposed by Sidoroff (1974) [26], has been deeply investigated by several other authors in the isotropic case, see for example [5,12-14,21] among other references. On the other hand, the present approach considers the continuum as a superposition of the matrix 
and further one-dimensional continua, each of them representing one family of fibres, as recently proposed by Klinkel, Sansour and Wagner (2005) [11] in the context of finite anisotropic elastoplasticity. The different continua are implicitly linked by the kinematical constraint that the deformation gradient together with its multiplicative split apply to all of them. In this formulation, the quantities obtained from the elastically relaxing part of the deformation gradient together with the structural tensor of each family of fibres are interpreted as a set of kinematical internal variables that are associated with the viscous motion of the material. Their evolution equations are obtained following standard arguments of the continuum thermodynamics where we postulate the existence of viscoelastic potentials for each compound in terms of their over-stresses (non-equilibrium stresses in the sense of non-equilibrium thermodynamics). This formulation differs from the approach by, for example, Holzapfel and Gasser [8].

From the continuum thermodynamics point of view, the additive split of the free energy function leads to separate constitutive equations for the matrix and for the fibres. Each constituant is then treated separately. On the one hand, as the matrix is considered as isotropic, one can then take advantage of the formulations and the algorithmic treatments deeply investigated in the recent literature. On the other hand, for the fibre bundles, a simple onedimensional viscoelastic modelling framework is developed. The corresponding scalar-valued evolution equations result from the positiveness of the reduced dissipation function for each family of fibres. The directional responses of the fibres induce then an anisotropic behaviour of the whole composite in the equilibrium and non-equilibrium thermodynamic regimes. The resulting framework is very similar to that of the anisotropic elastoplasticity at finite strains recently proposed by Klinkel, Sansour and Wagner [11]. In addition to its conceptual simplicity, the numerical effort is of the order of that devoted for an isotropic viscoelastic computation. Only a single-scalar valued resolution procedure is appended for each family of fibres, which results from the implicit integration of the scalar-valued evolution equation, and which renders the whole numerical procedure very efficient.

The paper is organized as follows. In section 2, the kinematics based on the multiplicative split of the deformation gradient is established. We define the basic kinematical quantities that enter in the definition of the general form of the free energy function. The constitutive relations together with the evolution equations are then derived in section 3 following the standard arguments of continuum thermodynamics. Model examples are proposed and discussed in section 4. In particular, we discuss issues concerning the fact that the fibres can carry load only in tension. Then, as far as the numerical approximation is concerned, a numerical design to implement the equations at hand is addressed within the context of the finite element method in section 5. A special attention is devoted to the (simple) determination of the algorithmic viscoelastic tangent 
moduli related to the fibres, and which enter in the composition of global tangent modulus within the Newton-Raphson iterative scheme. The efficiency of the proposed framework is then evaluated numerically through a set of representative numerical simulations in section 6 , and finally, conclusions are drawn in section 7 . Noteworthy remarks and comments are given throughout this paper.

\section{Basic kinematic assumptions}

As a point of departure, we establish in this section the kinematic assumptions that will be used throughout the following developments. The basic definitions follow closely those given by Klinkel, Sansour and Wagner [11] in anisotropic elastoplasticity. Before going on, and in all what follows, let $\boldsymbol{\varphi}(., t)$ denotes the motion in a time interval $[0, T]$ of a continuum body with reference configuration $\Omega_{0}$. The material particles are labeled by $\mathbf{X} \in \Omega_{0}$, and the deformation gradient is given by $\mathbf{F} \equiv \mathbf{F}(\mathbf{X}, t)=D \varphi(\mathbf{X}, t)$. This kinematics applies here to a solid which exhibits more than one constituent. In our specific case, we suppose that one of the constituents (the matrix) will always behave in an isotropic manner, and the other constituents will be defined as one-dimensional continua which can deform and carry loading only in their specific respective directions. The number of the families of fibres is not restricted, and each one-dimensional continuum will be associated with one individual family.

Let denote by $\vec{V}_{\mathrm{F}}$ the direction of a typical family of fibres in the reference configuration $\Omega_{0}\left(\vec{V}_{\mathrm{F}}\right.$ is a normalized vector). This latter transforms into the current configuration $\Omega_{t} \equiv \varphi\left(\Omega_{0}, t\right)$ by the map $\vec{v}_{\mathrm{F}}=\mathbf{F} \vec{V}_{\mathrm{F}}$ (here the vector $\vec{v}_{\mathrm{F}}$ is not normalized). On the one hand, the fact that the fibres behave in a one-dimensional manner necessitates the introduction of the corresponding one-dimensional deformation gradient as $\mathbf{F}_{\mathrm{F}}=\vec{v}_{\mathrm{F}} \otimes \vec{V}_{\mathrm{F}}$. 0n the other hand, the introduction of the structural tensor $\mathbf{M}_{\mathrm{F}}$ defined as $\mathbf{M}_{\mathrm{F}}=\vec{V}_{\mathrm{F}} \otimes \vec{V}_{\mathrm{F}}$, leads to the identity,

$$
\mathbf{F}_{\mathrm{F}}=\mathbf{F M}_{\mathrm{F}}
$$

That is, $\mathbf{F}_{\mathrm{F}}$ is no more than the projection of the deformation gradient in the direction of the associated family of fibres. Note also that the structural tensor $\mathbf{M}_{\mathrm{F}}$ is idempotent, i.e. $\mathbf{M}_{\mathrm{F}}^{n}=\mathbf{M}_{\mathrm{F}}$ for any value of the power $n$.

The approach to viscoelasticity starts with the multiplicative decomposition of the deformation gradient into a non-equilibrium elastic part $\mathbf{F}^{\mathrm{e}}$ and a viscous part $\mathbf{F}^{\mathrm{v}}$, 


$$
\mathbf{F}=\mathbf{F}_{\mathrm{M}}^{\mathrm{e}} \mathbf{F}_{\mathrm{M}}^{\mathrm{v}}
$$

where, due to the fact that this decomposition is assumed to be fully applied to the matrix, the index $\mathrm{M}$ is added. Here $\mathbf{F}_{\mathrm{M}}^{\mathrm{v}}$ is thought to be a kinematical internal variable associated with the viscous motion of the matrix. The variable $\mathbf{F}_{\mathrm{M}}^{\mathrm{e}}$ may be interpreted as the elastically relaxing portion of the matrix motion. It is implicitly defined for a given motion through (2) once $\mathbf{F}_{\mathrm{M}}^{\mathrm{v}}$ is known. And for the fibres, a similar multiplicative split is assumed for the one-dimensional deformation gradient $\mathbf{F}_{\mathrm{F}}$ :

$$
\mathbf{F}_{\mathrm{F}}=\mathbf{F}_{\mathrm{F}}^{\mathrm{e}} \mathbf{F}_{\mathrm{F}}^{\mathrm{v}} \text {. }
$$

For the matrix and for each fibre family, we define the following important kinematical quantities of the right Cauchy-Green type by the conventional relations:

$$
\begin{array}{lll}
\mathbf{C}=\mathbf{F}^{T} \mathbf{F}, & \mathbf{C}_{\mathrm{M}}^{\mathrm{e}}=\mathbf{F}_{\mathrm{M}}^{\mathrm{e}^{T}} \mathbf{F}_{\mathrm{M}}^{\mathrm{e}}, & \mathbf{C}_{\mathrm{M}}^{\mathrm{v}}=\mathbf{F}_{\mathrm{M}}^{\mathrm{v}^{T}} \mathbf{F}_{\mathrm{M}}^{\mathrm{v}}, \\
\mathbf{C}_{\mathrm{F}}=\mathbf{F}_{\mathrm{F}}^{T} \mathbf{F}_{\mathrm{F}}, & \mathbf{C}_{\mathrm{F}}^{\mathrm{e}}=\mathbf{F}_{\mathrm{F}}^{\mathrm{e}^{T}} \mathbf{F}_{\mathrm{F}}^{\mathrm{e}}, & \mathbf{C}_{\mathrm{F}}^{\mathrm{v}}=\mathbf{F}_{\mathrm{F}}^{\mathrm{v}^{T}} \mathbf{F}_{\mathrm{F}}^{\mathrm{v}} .
\end{array}
$$

Like for the purely isotropic viscoelasticity, see for example [21], the elastically relaxing right Cauchy-Green tensor $\mathbf{C}_{\mathrm{M}}^{\mathrm{e}}$ will enter in the definition of the nonequilibrium part of the free energy function relative to the matrix. A similar quantity must then be defined for the fibres. Let us first note that $\mathbf{F}_{\mathrm{F}}^{\mathrm{v}}$ must be of the one-dimensional form. We write then:

$$
\mathbf{F}_{\mathrm{F}}^{\mathrm{v}}=\lambda_{\mathrm{F}}^{\mathrm{v}} \mathbf{M}_{\mathrm{F}} \quad \Longrightarrow \quad \mathbf{C}_{\mathrm{F}}^{\mathrm{v}}=\lambda_{\mathrm{F}}^{\mathrm{v}^{2}} \mathbf{M}_{\mathrm{F}}
$$

with the initial condition relative to the reference state $\lambda_{\mathrm{F}}^{\mathrm{v}}(t=0)=1$. The kinematical (scalar) internal variable $\lambda_{\mathrm{F}}^{\mathrm{v}}$ is thought to be the viscous elongation associated to the individual fibre family under consideration.

Combining the relations $(3),(4)_{4}$ and $(5)_{1}$, the total right Cauchy-Green tensor relative to the fibres is rewritten as

$$
\mathbf{C}_{\mathrm{F}}=\lambda_{\mathrm{F}}^{\mathrm{v}^{2}} \mathbf{M}_{\mathrm{F}} \mathbf{F}_{\mathrm{F}}^{\mathrm{e}^{T}} \mathbf{F}_{\mathrm{F}}^{\mathrm{e}} \mathbf{M}_{\mathrm{F}}
$$

By analogy with the recent work in anisotropic elastoplasticity, see [11], the crucial idea is that the tensor $\mathbf{F}_{\mathrm{F}}^{\mathrm{e}} \mathbf{M}_{\mathrm{F}}$ defines an equivalent class of elastically relaxing parts of the fibre family's deformation gradient. Then, introducing the following definition for the class: 


$$
\widetilde{\mathbf{F}}_{\mathrm{F}}^{\mathrm{e}}=\mathbf{F}_{\mathrm{F}}^{\mathrm{e}} \mathbf{M}_{\mathrm{F}} \quad \Longrightarrow \quad \mathbf{F}_{\mathrm{F}}=\lambda_{\mathrm{F}}^{\mathrm{v}} \widetilde{\mathbf{F}}_{\mathrm{F}}^{\mathrm{e}},
$$

we define the corresponding elastically relaxing right Cauchy-Green tensor which is a representative for the non-equilibrium elastic measure for the fibres:

$$
\widetilde{\mathbf{C}}_{\mathrm{F}}^{\mathrm{e}}=\widetilde{\mathbf{F}}_{\mathrm{F}}^{\mathrm{e}^{T}} \widetilde{\mathbf{F}}_{\mathrm{F}}^{\mathrm{e}}=\frac{1}{\lambda_{\mathrm{F}}^{\mathrm{v}^{2}}} \mathbf{M}_{\mathrm{F}} \mathbf{C M} \mathbf{M}_{\mathrm{F}}
$$

This latter will enter in the definition of the non-equilibrium part of the free energy function relative to the fibres. The useful relation $\mathbf{C}_{\mathrm{F}}=\mathbf{M}_{\mathrm{F}} \mathbf{C M}_{\mathrm{F}}$ has been employed to get the relation (8).

\section{Constitutive equations and evolution laws}

To avoid any confusion in the following developments, only the kinematical quantities $\widetilde{\mathbf{F}}_{\mathrm{F}}^{\mathrm{e}}$ and $\widetilde{\mathbf{C}}_{\mathrm{F}}^{\mathrm{e}}$ representing the equivalent class for each family of fibres will be considered. Hence, for the sake of clarity, they will be denoted by $\mathbf{F}_{\mathrm{F}}^{\mathrm{e}}$ and $\mathbf{C}_{\mathrm{F}}^{\mathrm{e}}$, respectively. Confining our attention to the purely mechanical theory, the fundamental thermodynamic requirement can be expressed in terms of the isothermal Clausius-Duhem inequality as

$$
\mathcal{D}=\mathbf{S}: \frac{1}{2} \dot{\mathbf{C}}-\dot{\psi} \equiv \mathbf{\Sigma}: \mathbf{L}-\dot{\psi} \geq 0
$$

where $\mathbf{S}$ is the symmetric second Piola-Kirchhoff stress tensor, $\boldsymbol{\Sigma}$ is a material stress tensor defined as $\mathbf{\Sigma}=\mathbf{C S}$, and $\mathbf{L}=\mathbf{F}^{-1} \dot{\mathbf{F}}$ is the right rate of the (total) deformation gradient F. As in [15,24], $\boldsymbol{\Sigma}$ is an Eshelby-like stress tensor, since up to a sign and a spherical part it coincides with it. Note also that in [25], $\Sigma$ is called a Mandel-like stress tensor as the later is being defined with respect to the so-called intermediate configuration. Finally, $\psi$ is the free energy function which characterizes the state of the (composite) material and which has to be specified.

The non-equilibrium energetic contribution of each compound of the fibrereinforced material is assumed to depend on $\mathbf{F}_{\mathrm{M}}^{\mathrm{e}}$ and $\mathbf{F}_{\mathrm{F}}^{\mathrm{e}}$ through $\mathbf{C}_{\mathrm{M}}^{\mathrm{e}}$ and $\mathrm{C}_{\mathrm{F}}^{\mathrm{e}}$, respectively, such that the overall free energy function of the composite material can be expressed as

$$
\psi=\psi_{\infty_{M}}(\mathbf{C})+\psi_{\text {neq }_{M}}\left(\mathbf{C}_{M}^{e}\right)+\sum_{\mathrm{F}=1}^{\mathrm{nF}}\left\{\psi_{\infty_{\mathrm{F}}}\left(\mathbf{C}_{\mathrm{F}}\right)+\psi_{\mathrm{neq}_{\mathrm{F}}}\left(\mathbf{C}_{\mathrm{F}}^{\mathrm{e}}\right)\right\}
$$


where $\mathrm{nF}$ is the total number of the fibre families, $\psi_{\infty_{\mathrm{M}}}$ and $\psi_{\infty_{\mathrm{F}}}$ are the free energies at thermodynamic equilibrium for the matrix and for each fibres' family, respectively, and $\psi_{\text {neq }_{\mathrm{M}}}$ and $\psi_{\text {neq }_{\mathrm{F}}}$ are the corresponding non-equilibrium free energies. This functional assumption is necessary in order to satisfy frame invariance requirements. The expression (10) is motivated by the simple rheological model of the standard solid. That is, a free spring in parallel with a Maxwell element. Loosely speaking, $\mathbf{C}$ (resp. $\mathbf{C}_{\mathrm{F}}$ ) is the strain associated with the free spring, and $\mathbf{C}_{\mathrm{M}}^{\mathrm{e}}$ (resp. $\mathbf{C}_{\mathrm{F}}^{\mathrm{e}}$ ) is the strain associated with the one of the Maxwell element.

One the one hand, in taking the time derivative of the general free energy function (10), use is made of the following kinematical relations:

$$
\begin{aligned}
& \dot{\mathbf{C}}=\mathbf{L}^{T} \mathbf{C}+\mathbf{C L}, \\
& \dot{\mathbf{C}}_{\mathrm{M}}^{\mathrm{e}}=\mathbf{F}_{\mathrm{M}}^{\mathrm{v}^{-T}}\left\{\left(\mathbf{L}-\mathbf{L}_{\mathrm{M}}^{\mathrm{v}}\right)^{T} \mathbf{C}+\mathbf{C}\left(\mathbf{L}-\mathbf{L}_{\mathrm{M}}^{\mathrm{v}}\right)\right\} \mathbf{F}_{\mathrm{M}}^{\mathrm{v}^{-1}}, \\
& \dot{\mathbf{C}}_{\mathrm{F}}=\mathbf{M}_{\mathrm{F}}\left\{\mathbf{L}^{T} \mathbf{C}+\mathbf{C L}\right\} \mathbf{M}_{\mathrm{F}}, \\
& \dot{\mathbf{C}}_{\mathrm{F}}^{\mathrm{e}}=\frac{1}{\lambda_{\mathrm{F}}^{\mathrm{v}^{2}}} \mathbf{M}_{\mathrm{F}}\left\{\mathbf{L}^{T} \mathbf{C}+\mathbf{C L}-2 \frac{\dot{\lambda}_{\mathrm{F}}^{\mathrm{v}}}{\lambda_{\mathrm{F}}^{\mathrm{v}}} \mathbf{C}\right\} \mathbf{M}_{\mathrm{F}},
\end{aligned}
$$

where $\mathbf{L}_{\mathrm{M}}^{\mathrm{v}}=\mathbf{F}_{\mathrm{M}}^{\mathrm{v}^{-1}} \dot{\mathbf{F}}_{\mathrm{M}}^{\mathrm{v}}$ is the right rate of the viscous part of the deformation gradient. On the other hand, let us introduce the following definitions:

$$
\begin{array}{ll}
\boldsymbol{\Sigma}_{\infty_{\mathrm{M}}}=2 \mathbf{C} \frac{\partial \psi_{\infty_{\mathrm{M}}}}{\partial \mathbf{C}}, & \boldsymbol{\Sigma}_{\infty_{\mathrm{F}}}=2 \mathbf{C} \mathbf{M}_{\mathrm{F}} \frac{\partial \psi_{\infty_{\mathrm{F}}}}{\partial \mathbf{C}_{\mathrm{F}}} \mathbf{M}_{\mathrm{F}}, \\
\boldsymbol{\Sigma}_{\mathrm{neq}_{\mathrm{M}}}=2 \mathbf{C} \mathbf{F}_{\mathrm{M}}^{\mathrm{v}^{-1}} \frac{\partial \psi_{\mathrm{neq}_{\mathrm{M}}}}{\partial \mathbf{C}_{\mathrm{M}}^{\mathrm{e}}} \mathbf{F}_{\mathrm{M}}^{\mathrm{v}^{-T}}, & \boldsymbol{\Sigma}_{\mathrm{neq}_{\mathrm{F}}}=\frac{2}{\lambda_{\mathrm{F}}^{\mathrm{v}^{2}}} \mathbf{C} \mathbf{M}_{\mathrm{F}} \frac{\partial \psi_{\mathrm{neq}_{\mathrm{F}}}}{\partial \mathbf{C}_{\mathrm{F}}^{\mathrm{e}}} \mathbf{M}_{\mathrm{F}} .
\end{array}
$$

Then, inserting these results into the inequality (9) and using the standard arguments of continuum thermodynamics, see for example $[2,3]$, on obtains after some algebraic manipulations the following constitutive relation for the Eshelby-like stress tensor:

$$
\Sigma=\Sigma_{\infty_{\mathrm{M}}}+\Sigma_{\mathrm{neq}_{\mathrm{M}}}+\sum_{\mathrm{F}=1}^{\mathrm{nF}}\left\{\Sigma_{\infty_{\mathrm{F}}}+\Sigma_{\mathrm{neq}_{\mathrm{F}}}\right\},
$$

where $\boldsymbol{\Sigma}_{\infty_{\mathrm{M}}}$ and $\boldsymbol{\Sigma}_{\infty_{\mathrm{F}}}$ are the equilibrium parts concerning the matrix and the individual fibre families respectively, and where $\boldsymbol{\Sigma}_{\text {neq }}$ and $\boldsymbol{\Sigma}_{\text {neq }}$ are the corresponding non-equilibrium parts. The reduced dissipation (9) becomes then: 


$$
\mathcal{D}=\underbrace{\boldsymbol{\Sigma}_{\mathrm{neq}_{\mathrm{M}}}: \mathbf{L}_{\mathrm{M}}^{\mathrm{v}}}_{\mathcal{D}_{\mathrm{M}}}+\sum_{\mathrm{F}=1}^{\mathrm{nF}}\{\underbrace{\Sigma_{\text {neq }_{\mathrm{F}}}: \mathbf{M}_{\mathrm{F}} \frac{\dot{\lambda}_{\mathrm{F}}^{\mathrm{v}}}{\lambda_{\mathrm{F}}^{\mathrm{v}}}}_{\mathcal{D}_{\mathrm{F}}}\} \geq 0 .
$$

This latter must be satisfied for all admissible processes in the material. This restriction could in turn be satisfied by imposing separately the positiveness of the dissipation in the matrix, $\mathcal{D}_{\mathrm{M}} \geq 0$, and in each family of fibres, $\mathcal{D}_{\mathrm{F}} \geq 0$.

Remark 1 The present model can be easily extended to the case of multiple relaxation mechanisms by assuming that there exists a set of internal variables $\mathbf{F}^{\mathbf{v}^{k}}(k=1 \ldots N)$ such that, instead of (2), one has

$$
\mathbf{F}=\mathbf{F}_{\mathrm{M}}^{\mathrm{e}^{k}} \mathbf{F}_{\mathrm{M}}^{\mathrm{v}^{k}} \quad k=1 \ldots N
$$

In our case, this split will be applied to all the compounds of the fibre-reinforced material. Here the variables $\mathbf{F}_{\mathrm{M}}^{\mathrm{v}^{k}}$ can be interpreted as the viscous portion of the motion associated with the individual relaxation mechanisms. See [5] for more details in the isotropic case.

\subsection{Stress tensor: the matrix contribution}

Concerning the matrix contribution to the total stress tensor, the constitutive relations $(12)_{1}$ and $(12)_{3}$ can be rewritten in the following equivalent forms:

$$
\mathbf{S}_{\infty_{\mathrm{M}}}=2 \frac{\partial \psi_{\infty_{\mathrm{M}}}}{\partial \mathbf{C}} \quad \text { and } \quad \mathbf{S}_{\mathrm{neq}_{\mathrm{M}}}=2 \mathbf{F}_{\mathrm{M}}^{\mathrm{v}^{-1}} \frac{\partial \psi_{\mathrm{neq}_{\mathrm{M}}}}{\partial \mathbf{C}_{\mathrm{M}}^{\mathrm{e}}} \mathbf{F}_{\mathrm{M}}^{\mathrm{v}^{-T}}
$$

where $\mathbf{S}_{\infty_{\mathrm{M}}}=\mathbf{C}^{-1} \boldsymbol{\Sigma}_{\infty_{\mathrm{M}}}$ and $\mathbf{S}_{\mathrm{neq}_{\mathrm{M}}}=\mathbf{C}^{-1} \boldsymbol{\Sigma}_{\text {neq }}$ are respectively the equilibrium and non-equilibrium second Piola-Kirchhoff stress tensors associated with the matrix. Note that as this latter is considered isotropic, this restriction is assumed at the reference configuration and on the intermediate one defined by $\mathbf{F}_{\mathrm{M}}^{\mathrm{v}}$. Hence, the part $\psi_{\infty_{\mathrm{M}}}$ of the free energy function depends on the invariants of $\mathbf{C}$ which are identical to those of the (total) left Cauchy-Green tensor $\mathbf{b}=\mathbf{F F}^{T}$. Similarly, the part $\psi_{\text {neq }_{\mathrm{M}}}$ of the free energy function depends on the invariants of $\mathbf{C}_{\mathrm{M}}^{\mathrm{e}}$ which are identical to those of the corresponding elastically relaxing left Cauchy-Green tensor $\mathbf{b}_{\mathrm{M}}^{\mathrm{e}}=\mathbf{F}_{\mathrm{M}}^{\mathrm{e}} \mathbf{F}_{\mathrm{M}}^{\mathrm{e}^{T}}$. Then, from the well-known result of isotropic function theory, see for example [7,16,33], the corresponding equilibrium and non-equilibrium Kirchhoff stress tensors $\boldsymbol{\tau}_{\infty_{\mathrm{M}}}=\mathbf{F S}_{\infty_{\mathrm{M}}} \mathbf{F}^{T}$ and $\tau_{\text {neq }_{\mathrm{M}}}=\mathbf{F S}_{\text {neq }_{\mathrm{M}}} \mathbf{F}^{T}$ can be equivalently given by, 


$$
\boldsymbol{\tau}_{\infty_{\mathrm{M}}}=2 \frac{\partial \psi_{\infty_{\mathrm{M}}}}{\partial \mathbf{b}} \mathbf{b} \quad \text { and } \quad \boldsymbol{\tau}_{\mathrm{neq}_{\mathrm{M}}}=2 \frac{\partial \psi_{\mathrm{neq}_{\mathrm{M}}}}{\partial \mathbf{b}_{\mathrm{M}}^{\mathrm{e}}} \mathbf{b}_{\mathrm{M}}^{\mathrm{e}}
$$

\subsection{Stress tensor: the fibres' contribution}

Concerning the fibres, the following expressions for the equilibrium and nonequilibrium (one-dimensional) second Piola-Kirchhoff stress tensors can be deduced from the constitutive relations $(12)_{2}$ and $(12)_{4}$, respectively:

$$
\mathbf{S}_{\infty_{\mathrm{F}}}=\underbrace{2\left[\frac{\partial \psi_{\infty_{\mathrm{F}}}}{\partial \mathbf{C}_{\mathrm{F}}}: \mathbf{M}_{\mathrm{F}}\right]}_{=\sigma_{\infty_{\mathrm{F}}}} \mathbf{M}_{\mathrm{F}} \text { and } \mathbf{S}_{\mathrm{neq}_{\mathrm{F}}}=\underbrace{\frac{2}{\lambda_{\mathrm{F}}^{\mathrm{v}^{2}}}\left[\frac{\partial \psi_{\mathrm{neq}_{\mathrm{F}}}}{\partial \mathbf{C}_{\mathrm{F}}^{e}}: \mathbf{M}_{\mathrm{F}}\right]}_{=\sigma_{\mathrm{neq}_{\mathrm{F}}}} \mathbf{M}_{\mathrm{F}} .
$$

These expressions render the treatment of the fibres' contribution to the global behaviour very attractive since the structural tensor $\mathbf{M}_{\mathrm{F}}$ is a fixed known quantity relative to the reference configuration, and where $\sigma_{\infty_{\mathrm{F}}}$ and $\sigma_{\text {neq }}$ are simply stress-like scalar quantities.

\subsection{Local evolution equations}

For the matrix component, the condition $\mathcal{D}_{M} \geq 0$ issued from the reduced inequality (14) is nowadays well-known in finite isotropic inelasticity, see for example $[7,21,28]$ among others. It is expressible in the spatial configuration in the following equivalent form:

$$
\mathcal{D}_{\mathrm{M}}=\tau_{\mathrm{neq}_{\mathrm{M}}}:\left[-\frac{1}{2}\left(£_{\mathrm{v}} \mathbf{b}_{\mathrm{M}}^{\mathrm{e}}\right) \mathbf{b}_{\mathrm{M}}^{\mathrm{e}^{-1}}\right] \geq 0,
$$

where $£_{\mathrm{v}} \mathbf{b}_{\mathrm{M}}^{\mathrm{e}}$ is the Lie derivative of $\mathbf{b}_{\mathrm{M}}^{\mathrm{e}}=\mathbf{F C}_{\mathrm{M}}^{\mathrm{v}^{-1}} \mathbf{F}^{T}$ defined as

$$
£_{\mathrm{v}} \mathbf{b}_{\mathrm{M}}^{\mathrm{e}}=\mathbf{F} \frac{\mathrm{d}}{\mathrm{d} t}\left[\mathbf{C}_{\mathrm{M}}^{\mathrm{v}^{-1}}\right] \mathbf{F}^{T}
$$

For the evolution equation of the internal variable $\mathbf{b}_{\mathrm{M}}^{\mathrm{e}}$, we assume the existence of a viscoelastic potential (a creep-like potential) $\phi_{\mathrm{vis}_{\mathrm{M}}}\left(\boldsymbol{\tau}_{\mathrm{neq}_{\mathrm{M}}}\right)$, i.e. a convex and positive function with $\phi_{\mathrm{vis}_{\mathrm{M}}}=0 \Leftrightarrow \boldsymbol{\tau}_{\mathrm{neq}_{\mathrm{M}}}=\mathbf{0}$, such that 


$$
-\frac{1}{2}\left(£_{\mathrm{v}} \mathbf{b}_{\mathrm{M}}^{\mathrm{e}}\right) \mathbf{b}_{\mathrm{M}}^{\mathrm{e}^{-1}}=\frac{\partial \phi_{\mathrm{vis}_{\mathrm{M}}}}{\partial \boldsymbol{\tau}_{\mathrm{neq}_{\mathrm{M}}}} \quad \Longrightarrow \quad £_{\mathrm{v}} \mathbf{b}_{\mathrm{M}}^{\mathrm{e}}=-2 \frac{\partial \phi_{\mathrm{vis}_{\mathrm{M}}}}{\partial \boldsymbol{\tau}_{\mathrm{neq}_{\mathrm{M}}}} \mathbf{b}_{\mathrm{M}}^{\mathrm{e}}
$$

Hence, the convexity of $\phi_{\mathrm{vis}_{\mathrm{M}}}$ is then sufficient to satisfy the inequality (19) for any admissible process. Note that such an assumption has already been made in, for example, [4,21], and in [17] within the context of finite isotropic viscoelastic-plasticity.

Now for the fibres, the treatment of the evolution equations is new. For each family, the following condition must be satisfied (see (14)):

$$
\mathcal{D}_{\mathrm{F}}=\boldsymbol{\Sigma}_{\mathrm{neq}_{\mathrm{F}}}: \mathbf{M}_{\mathrm{F}} \frac{\dot{\lambda}_{\mathrm{F}}^{\mathrm{v}}}{\lambda_{\mathrm{F}}^{\mathrm{v}}} \geq 0
$$

Here again we assume the existence of a viscoelastic potential $\phi_{\mathrm{vis}_{\mathrm{F}}}\left(\boldsymbol{\Sigma}_{\mathrm{neq}_{\mathrm{F}}}\right)$, expressed in the reference configuration, such that

$$
\mathbf{M}_{\mathrm{F}} \frac{\dot{\lambda}_{\mathrm{F}}^{\mathrm{v}}}{\lambda_{\mathrm{F}}^{\mathrm{v}}}=\frac{\partial \phi_{\mathrm{vis}_{\mathrm{F}}}}{\partial \boldsymbol{\Sigma}_{\mathrm{neq}_{\mathrm{F}}}} \quad \Longrightarrow \quad \dot{\lambda}_{\mathrm{F}}^{\mathrm{v}} \mathbf{M}_{\mathrm{F}}=\lambda_{\mathrm{F}}^{\mathrm{v}} \frac{\partial \phi_{\mathrm{vis}_{\mathrm{F}}}}{\partial \boldsymbol{\Sigma}_{\mathrm{neq}_{\mathrm{F}}}}
$$

For the following examples, we choose for this potential a simple quadratic expression given by

$$
\phi_{\mathrm{vis}_{\mathrm{F}}}\left(\boldsymbol{\Sigma}_{\mathrm{neq}_{\mathrm{F}}}\right)=\frac{1}{2 \eta_{\mathrm{F}}}\left(\mathbf{M}_{\mathrm{F}} \boldsymbol{\Sigma}_{\mathrm{neq}_{\mathrm{F}}} \mathbf{M}_{\mathrm{F}}: \mathbf{M}_{\mathrm{F}} \boldsymbol{\Sigma}_{\mathrm{neq}_{\mathrm{F}}} \mathbf{M}_{\mathrm{F}}\right)
$$

where $\eta_{\mathrm{F}}$ is a viscosity parameter corresponding to the fibres. The equation $(23)_{2}$ reduces then to the following single scalar-valued evolution equation of the internal variable $\lambda_{\mathrm{F}}^{\mathrm{v}}$ :

$$
\dot{\lambda}_{\mathrm{F}}^{\mathrm{v}}=\frac{\lambda_{\mathrm{F}}^{\mathrm{v}}}{\eta_{\mathrm{F}}} \sigma_{\mathrm{neq}_{\mathrm{F}}}\left[\mathbf{C}: \mathbf{M}_{\mathrm{F}}\right]
$$

where the non-equilibrium stress-like scalar variable $\sigma_{\text {neq }_{\mathrm{F}}}$ has been defined in $(18)_{2}$. To obtain this latter equation, use has been made of the relation $\boldsymbol{\Sigma}_{\mathrm{neq}}=\mathbf{C S}_{\mathrm{neq}_{\mathrm{F}}} \equiv \sigma_{\mathrm{neq}_{\mathrm{F}}} \mathbf{C M}_{\mathrm{F}}$, the idempotent property $\mathbf{M}_{\mathrm{F}}^{n}=\mathbf{M}_{\mathrm{F}}$ for every power $n$, and the useful identity $\mathbf{M}_{\mathrm{F}} \mathbf{C M}_{\mathrm{F}}=\left[\mathbf{C}: \mathbf{M}_{\mathrm{F}}\right] \mathbf{M}_{\mathrm{F}}$. 


\section{Anisotropic viscoelastic model examples}

To make matters as concrete as possible, we give in this section model examples within the present anisotropic formulation with a special focus on the fibres's behaviour. However, before that, we first choose a constitutive model for the matrix that will be used in our applications. As this latter is isotropic, its behaviour can be described by any of the existing models in the literature. For the equilibrium part of the free energy function $\psi_{\infty_{M}}$, we choose the following Ogden's hyperelastic model [19], see also [30]:

$$
\psi_{\infty_{\mathrm{M}}}(\mathbf{C})=\kappa_{\mathrm{M}}(J-\log [J]-1)+\mu_{\mathrm{M}} \sum_{A=1,3} \sum_{\alpha=1,3} \frac{c_{\alpha}}{m_{\alpha}^{2}}\left[\bar{\lambda}_{A}^{m_{\alpha}}-1\right]
$$

where $J=\operatorname{det}[\mathbf{F}]=(\operatorname{det}[\mathbf{C}])^{1 / 2}, \bar{\lambda}_{A}=J^{-1 / 3} \lambda_{A}\left(\left\{\lambda_{A}\right\}_{A=1,2,3}\right.$ being the principal (total) stretches corresponding to $\mathbf{C}$, or $\mathbf{b}$ ), $\kappa_{\mathrm{M}}>0$ and $\mu_{\mathrm{M}}>0$ are the matrix bulk and shear moduli at equilibrium, respectively, and where $\left\{c_{\alpha}, m_{\alpha}\right\}_{\alpha=1,2,3}$ are the Ogden's parameters. And for the non-equilibrium part of the free energy function $\psi_{\text {neq }_{\mathrm{M}}}$, we consider for simplicity the following Hencky-type model also given in terms of principal stretches:

$$
\psi_{\text {neq }_{\mathrm{M}}}\left(\mathbf{C}_{\mathrm{M}}^{\mathrm{e}}\right)=\frac{1}{2} \kappa_{\mathrm{neq}_{\mathrm{M}}}\left(\log \left[J_{\mathrm{M}}^{\mathrm{e}}\right]\right)^{2}+\mu_{\mathrm{neq}_{\mathrm{M}}} \sum_{A=1,3}\left(\log \left[\bar{\lambda}_{\mathrm{M}_{A}}^{\mathrm{e}}\right]\right)^{2}
$$

where $J_{\mathrm{M}}^{\mathrm{e}}=\operatorname{det}\left[\mathbf{F}_{\mathrm{M}}^{\mathrm{e}}\right]=\left(\operatorname{det}\left[\mathbf{C}_{\mathrm{M}}^{\mathrm{e}}\right]\right)^{1 / 2}, \bar{\lambda}_{\mathrm{M}_{A}}^{\mathrm{e}}=J_{\mathrm{M}}^{\mathrm{e}^{-1 / 3}} \lambda_{\mathrm{M}_{A}}^{\mathrm{e}}\left(\left\{\lambda_{\mathrm{M}_{A}}^{\mathrm{e}}\right\}_{A=1,2,3}\right.$ are the elastically relaxing principal stretches corresponding to $\mathbf{C}_{\mathrm{M}}^{\mathrm{e}}$, or $\left.\mathbf{b}_{\mathrm{M}}^{\mathrm{e}}\right), \kappa_{\text {neq }}>0$ and $\mu_{\text {neq }_{\mathrm{M}}}>0$ are the non-equilibrium bulk and shear moduli, respectively. Finally, for the viscoelastic potential $\phi_{\mathrm{vis}_{\mathrm{M}}}$ (see the evolution equation $(21)_{2}$ ), we choose the following quadratic form as already used in other references, see for example $[4,17,21]$ :

$$
\phi_{\mathrm{vis}_{\mathrm{M}}}\left(\boldsymbol{\tau}_{\mathrm{neq}_{\mathrm{M}}}\right)=\frac{1}{2 \eta_{\mathrm{d}_{\mathrm{M}}}} \operatorname{dev}\left[\boldsymbol{\tau}_{\mathrm{neq}_{\mathrm{M}}}\right]: \operatorname{dev}\left[\boldsymbol{\tau}_{\mathrm{neq}_{\mathrm{M}}}\right]+\frac{1}{9 \eta_{\mathrm{v}_{\mathrm{M}}}}\left(\boldsymbol{\tau}_{\mathrm{neq}_{\mathrm{M}}}: \mathbf{1}\right)^{2}
$$

where $\eta_{\mathrm{v}_{\mathrm{M}}}$ and $\eta_{\mathrm{d}_{\mathrm{M}}}$ are respectively the volumetric and the deviatoric viscosity parameters of the matrix. In (28), $\operatorname{dev}[$.$] is the deviatoric operator and \mathbf{1}$ is the second order identity tensor.

Remark 2 With the definitions (26)-(28), the volumetric and the isochoric contributions of the matrix are described separately. A quasi-incompressible (or isochoric) behaviour in the equilibrium and/or non-equilirium phases can be reached for relatively high values of the volumetric paramaters, i.e. $\kappa_{\mathrm{M}}$, $\kappa_{\mathrm{neq}_{\mathrm{M}}}$ and $\eta_{\mathrm{v}_{\mathrm{M}}}$. Also, notice that many other choices are also possible for these 
functions. For instance, instead of the Ogden's equilibrium free function (26), one can use a simpler one like for example a Hencky model of the type given by (27), or the nowadays classical Neo-Hookean model.

For the fibres' contribution, we present in the next sections two constitutive model examples among many other possibilities: a logarithmic model and a quadratic model.

\subsection{A logarithmic model for the fibres}

For the equilibrium part, we choose a similar constitutive equation as the one given by Klinkel, Sansour and Wagner in elastoplasticity [11]. The expression of the function $\psi_{\infty_{\mathrm{F}}}$ is defined as

$$
\psi_{\infty_{\mathrm{F}}}\left(\mathbf{C}_{\mathrm{F}}\right)=\frac{E_{\mathrm{F}}}{4}\left(I_{4} \log \left[I_{4}\right]-I_{4}+1\right)
$$

where $E_{\mathrm{F}}$ is the equilibrium Young's modulus of the fibre-continuum, and where $I_{4}=\mathbf{C}_{\mathrm{F}}: \mathbf{1} \equiv \mathbf{C}: \mathbf{M}_{\mathrm{F}}$ is no more than the fourth pseudo-invariant of the so-called integrity basis as proposed by Spencer (1984) [31], and nowadays widely employed for the modelling of purely elastic fibre-reinforced materials, see for example $[1,6,7,34]$. For the one-dimensional equilibrium second PiolaKirchhoff stress tensor $\mathbf{S}_{\infty_{\mathrm{F}}}=\sigma_{\infty_{\mathrm{F}}} \mathbf{M}_{\mathrm{F}}$ as defined in (18) $)_{1}$, the following simple form is obtained:

$$
\sigma_{\infty_{\mathrm{F}}}=\frac{E_{\mathrm{F}}}{2} \log \left[I_{4}\right]
$$

And for the non-equilibrium contribution of the fibres, we choose a similar expression for the corresponding free energy function:

$$
\psi_{\text {neq }_{\mathrm{F}}}\left(\mathbf{C}_{\mathrm{F}}^{\mathrm{e}}\right)=\frac{E_{\mathrm{neq}}}{4} \lambda_{\mathrm{F}}^{\mathrm{v}^{2}}\left(I_{4}^{\mathrm{e}} \log \left[I_{4}^{\mathrm{e}}\right]-I_{4}^{\mathrm{e}}+1\right),
$$

where $E_{\text {neq }}$ is the non-equilibrium Young's modulus of the fibre-continuum, and where $I_{4}^{\mathrm{e}}=\mathrm{C}_{\mathrm{F}}^{\mathrm{e}}: \mathbf{1}=I_{4} / \lambda_{\mathrm{F}}^{\mathrm{v}^{2}}$. For the corresponding one-dimensional nonequilibrium second Piola-Kirchhoff stress tensor $\mathbf{S}_{\text {neq }}=\sigma_{\text {neq }} \mathbf{M}_{\mathrm{F}}$ as defined in $(18)_{2}$, we obtain:

$$
\sigma_{\mathrm{neq}_{\mathrm{F}}}=\frac{E_{\mathrm{neq}_{\mathrm{F}}}}{2}\left(\log \left[I_{4}\right]-2 \log \left[\lambda_{\mathrm{F}}^{\mathrm{v}}\right]\right) .
$$


Note that this latter is in turn employed to describe the evolution of the internal variable $\lambda_{\mathrm{F}}^{\mathrm{v}}$ through the scalar-valued local evolution equation (25).

\subsection{A quadratic model for the fibres}

As the definitions of the fibres' parameters given in this model are identical to those of the logarithmic model, we give here directly the expressions of the characteristic functions. For the equilibrium and non-equilibrium parts of the free energy function we choose:

$$
\psi_{\infty_{\mathrm{F}}}\left(\mathbf{C}_{\mathrm{F}}\right)=\frac{E_{\mathrm{F}}}{8}\left(I_{4}-1\right)^{2}, \quad \psi_{\mathrm{neq}_{\mathrm{F}}}\left(\mathbf{C}_{\mathrm{F}}^{\mathrm{e}}\right)=\frac{E_{\mathrm{neq}_{\mathrm{F}}}}{8} \lambda_{\mathrm{F}}^{\mathrm{v}^{4}}\left(I_{4}^{\mathrm{e}}-1\right)^{2} .
$$

Then for the equilibrium and non-equilibrium second Piola-Kirchhoff stress components, we obtain respectively the following simple expressions:

$$
\sigma_{\infty_{\mathrm{F}}}=\frac{E_{\mathrm{F}}}{2}\left(I_{4}-1\right), \quad \sigma_{\mathrm{neq}_{\mathrm{F}}}=\frac{E_{\mathrm{neq}_{\mathrm{F}}}}{2}\left(I_{4}-\lambda_{\mathrm{F}}^{\mathrm{v}^{2}}\right) .
$$

Remark 3 Of course, other models for the fibres can be proposed. We think for example of an exponential model, similar to that employed by Holzapfel and Gasser (2001) [8], such that $\sigma_{\infty_{\mathrm{F}}}=E_{\mathrm{F}} \exp \left[I_{4}-1\right]$ and $\sigma_{\text {neq }_{\mathrm{F}}}=E_{\text {neq }_{\mathrm{F}}} \exp \left[I_{4}-\right.$

$\left.\lambda_{\mathrm{F}}^{\mathrm{v}^{2}}\right]$. Note that for equal parameters, i.e. $E_{\mathrm{F}}, E_{\mathrm{neq}_{\mathrm{F}}}$ and $\eta_{\mathrm{F}}$, this latter will exhibit a stiffer behaviour than the quadratic model, which in turn is stiffer than the logarithmic one.

\subsection{The no-compression behaviour of the fibres}

In general, the fibres can carry load only in tension. To take into account this important fact, the precedent fibre models can still be easily adapted. For the equilibrium elastic contribution, the pseudo-invariant $I_{4}=\mathbf{C}_{\mathrm{F}}: \mathbf{1}$ is no more than the square of the fibres' total stretch:

$$
I_{4}=\mathbf{C}: \mathbf{M}_{\mathrm{F}}=\mathbf{C}: \vec{V}_{\mathrm{F}} \otimes \vec{V}_{\mathrm{F}} \equiv \vec{v}_{\mathrm{F}} \cdot \vec{v}_{\mathrm{F}},
$$

where we recall that the spatial vector $\vec{v}_{\mathrm{F}}$ is not normalized. Then, $I_{4}>1$ corresponds to an extension of the fibres, while $I_{4}<1$ corresponds to their contraction. Hence, to take into account the unilateral behaviour of the fibres, the expressions of the longitudinal components of the equilibrium stresses for 
the logarithmic model (30) and for the quadratic one $(34)_{1}$ can for instance be respectively replaced by the following expressions:

$$
\sigma_{\infty_{\mathrm{F}}}=\frac{E_{\mathrm{F}}}{2}\left\langle\log \left[I_{4}\right]\right\rangle \quad \text { and } \quad \sigma_{\infty_{\mathrm{F}}}=\frac{E_{\mathrm{F}}}{2}\left\langle I_{4}-1\right\rangle
$$

where the Macauley bracket $\langle$.$\rangle defines the positive part function as \langle x\rangle=$ $\frac{1}{2}[x+|x|]$. Similarly for the non-equilibrium contribution to the behaviour, we can impose the restriction that $I_{4}^{\mathrm{e}} \geq 1$. Then the longitudinal components for the logarithmic model (32) and for the quadratic one $(34)_{2}$ can then be respectively replaced by,

$$
\sigma_{\mathrm{neq}_{\mathrm{F}}}=\frac{E_{\mathrm{neq}_{\mathrm{F}}}}{2}\left\langle\log \left[I_{4}\right]-2 \log \left[\lambda_{\mathrm{F}}^{\mathrm{v}}\right]\right\rangle \quad \text { and } \quad \sigma_{\mathrm{neq}_{\mathrm{F}}}=\frac{E_{\mathrm{neq}_{\mathrm{F}}}}{2}\left\langle I_{4}-\lambda_{\mathrm{F}}^{\mathrm{v}^{2}}\right\rangle .
$$

Remark 4 As the positive part function $\langle x\rangle$ is not differentiable, it is replaced by a smooth function $\langle x\rangle \equiv x \widetilde{H}(x)$ during the finite element implementation to avoid numerical difficulties. Here $\widetilde{H}(x)$ is a homographic approximation of the Heaviside function given by

$$
H(x)=\frac{1}{2}\left[1+\frac{x}{|x|}\right] \quad \longrightarrow \quad \widetilde{H}(x)=\frac{1}{2}\left[1+\frac{x}{|x|+\delta}\right]
$$

where $\delta>0$ is a small numerical parameter $\left(10^{-3}\right.$ or $10^{-4}$ in practice).

\section{Numerical approximation and tangent moduli}

For the actual implementation within the finite element method, the problem is solved herein by means of the Newton-Raphson iterative scheme. Accordingly, this requires the linearization of the weak form of the balance equation. One can then compute the tangent stiffness matrix of the discrete problem at each iteration through the interpolation functions. As for standard problems in the finite strains range, this matrix is the sum of a geometrical and a material contributions. The material contribution depends strongly on the adopted constituve model for the continuum and will be the focus of this section. 


\subsection{Numerical integration of the local evolution equations}

Concerning the viscoelastic evolution in the matrix, the resolution algorithm is identical to those adopted for purely isotropic materials. Hence, in this work, the local evolution equation $(21)_{2}$ is numerically solved by an implicit exponential scheme. See for example $[18,21]$ for more details.

For each family of fibres, the local evolution equation (25) is conveniently rewritten in the following form:

$$
\dot{\lambda}_{\mathrm{F}}^{\mathrm{v}}=\frac{\lambda_{\mathrm{F}}^{\mathrm{v}}}{\eta_{\mathrm{F}}} \sigma_{\mathrm{neq}_{\mathrm{F}}}\left(I_{4}, \lambda_{\mathrm{F}}^{\mathrm{v}}\right) I_{4}
$$

where the non-equilibrium stress component $\sigma_{\text {neq }}$ is a function which depends on the pseudo-invariant $I_{4}=\mathbf{C}: \mathbf{M}_{\mathrm{F}}$ and the unknown internal variable $\lambda_{\mathrm{F}}^{\mathrm{v}}$ either for the logarithmic model (see (32) or $(37)_{1}$ ) or for the quadratic one (see $(34)_{2}$ or $\left.(37)_{2}\right)$. During the numerical implementation within the finite element context, this equation must be solved locally at the integration points level for fixed increments of the deformation. Hence, using the implicit backward Euler scheme, this latter is approximated as

$$
\lambda_{\mathrm{F}_{n+1}}^{\mathrm{v}}-\lambda_{\mathrm{F}_{n}}^{\mathrm{v}}=\Delta t \frac{\lambda_{\mathrm{F}_{n+1}}^{\mathrm{v}}}{\eta_{\mathrm{F}}} \sigma_{\mathrm{neq}_{\mathrm{F}}}\left(I_{4}, \lambda_{\mathrm{F}_{n+1}}^{\mathrm{v}}\right) I_{4}
$$

where $\Delta t=t_{n+1}-t_{n}$ is the time increment, $\lambda_{\mathrm{F}_{n}}^{\mathrm{v}}$ is the known value of $\lambda_{\mathrm{F}}^{\mathrm{v}}$ at time $t=t_{n}$, and where $I_{4}$ is fixed during this local step. The scalar-valued equation (40) can be easily solved for $\lambda_{\mathrm{F}_{n+1}}^{\mathrm{v}}$ through a simple Newton iterative procedure as summarized in table 1.

\subsection{Algorithmic tangent moduli}

From the constitutive relation (13), the total second Piola-Kirchhoff stress tensor $\mathbf{S}=\mathbf{C}^{-1} \boldsymbol{\Sigma}$ and the total Kirchhoff stress tensor $\tau=\mathbf{F S F}^{T}=\mathbf{F}^{-T} \boldsymbol{\Sigma} \mathbf{F}^{T}$ are given by,

$$
\mathbf{S}=\mathbf{S}_{\infty_{\mathrm{M}}}+\mathbf{S}_{\mathrm{neq}_{\mathrm{M}}}+\sum_{\mathrm{F}=1}^{\mathrm{nF}}\left\{\mathbf{S}_{\infty_{\mathrm{F}}}+\mathbf{S}_{\mathrm{neq}_{\mathrm{F}}}\right\}, \quad \boldsymbol{\tau}=\boldsymbol{\tau}_{\infty_{\mathrm{M}}}+\boldsymbol{\tau}_{\mathrm{neq}_{\mathrm{M}}}+\sum_{\mathrm{F}=1}^{\mathrm{nF}}\left\{\boldsymbol{\tau}_{\infty_{\mathrm{F}}}+\boldsymbol{\tau}_{\mathrm{neq}_{\mathrm{F}}}\right\}
$$


Table 1

Local Newton iterative scheme to solve the fibres' viscoelastic evolution equation.

1. Initialize: $k=0, \quad \lambda_{\mathrm{F}_{n+1}}^{\mathrm{v}^{(0)}}=\lambda_{\mathrm{F}_{n}}^{\mathrm{v}}$

2. Evaluate the residual and check the convergence:

$$
\begin{gathered}
\sigma_{\text {neq }_{\mathrm{F}}}^{(k)}=\sigma_{\text {neq }_{\mathrm{F}}}\left(I_{4}, \lambda_{\mathrm{F}_{n+1}}^{\mathrm{v}^{(k)}}\right) \\
r^{(k)}=-\left(\lambda_{\mathrm{F}_{n+1}}^{\mathrm{v}^{(k)}}-\lambda_{\mathrm{F}_{n}}^{\mathrm{v}}\right)+\Delta t \frac{I_{4}}{\eta_{\mathrm{F}}} \lambda_{\mathrm{F}_{n+1}}^{\mathrm{v}^{(k)}} \sigma_{\text {neq }_{\mathrm{F}}}^{(k)} \\
\text { IF }\left|r^{(k)}\right|>\text { TOL, go to step 3. ELSE, } \lambda_{\mathrm{F}_{n+1}}^{\mathrm{v}}=\lambda_{\mathrm{F}_{n+1}}^{\mathrm{v}^{(k)}}, \text { EXIT. }
\end{gathered}
$$

3. Compute the derivative and increment:

$$
\begin{aligned}
& D^{(k)}=\left\{1-\Delta t \frac{I_{4}}{\eta_{\mathrm{F}}}\left[\sigma_{\text {neq }_{\mathrm{F}}^{(k)}}^{(k)}+\lambda_{\mathrm{F}_{n+1}}^{\mathrm{v}^{(k)}} \frac{\partial \sigma_{\text {neq }_{\mathrm{F}}}^{(k)}}{\partial \lambda_{\mathrm{F}}^{\mathrm{v}}}\right]\right\} \\
& \lambda_{\mathrm{F}_{n+1}}^{\mathrm{v}^{(k+1)}}=\lambda_{\mathrm{F}_{n+1}}^{\mathrm{v}^{(k)}}+\frac{r^{(k)}}{D^{(k)}}
\end{aligned}
$$

Set $k \longleftarrow k+1$ and return to Step 2 .

Hence, as the stress tensors are additively split, the corresponding tangent moduli are also additively split as

$$
\widetilde{\mathbf{C}}=\widetilde{\mathbf{C}}_{\mathrm{M}}+\sum_{\mathrm{F}=1}^{\mathrm{nF}} \widetilde{\mathbf{C}}_{\mathrm{F}} \quad \text { and } \quad \widetilde{\mathbf{C}}=\widetilde{\mathbf{C}}_{\mathrm{M}}+\sum_{\mathrm{F}=1}^{\mathrm{nF}} \widetilde{\mathbf{C}}_{\mathrm{F}}
$$

Here $\widetilde{\mathbf{C}}$ is the material tangent modulus. It is additively decomposed into a matrix contribution $\widetilde{\mathbf{C}}_{M}$ (the term associated with the sum $\mathbf{S}_{\infty_{M}}+\mathbf{S}_{\mathrm{neq}_{\mathrm{M}}}$ ) and the contribution of each family of fibres $\widetilde{\mathbf{C}}_{\mathrm{F}}$ (the term associated with the sum $\mathbf{S}_{\infty_{\mathrm{F}}}+\mathbf{S}_{\text {neq }_{\mathrm{F}}}$ ). Similarly, $\widetilde{\mathbf{C}}$ is the spatial tangent modulus decomposed into the contribution $\widetilde{\mathbf{C}}_{\mathrm{M}}$ relative to the matrix, and contributions $\widetilde{\mathbf{C}}_{\mathrm{F}}$ relative to each family of fibres. For a detailed computation of the isotropic matrix contribution to the tangent moduli, we refer to $[18,21]$ among others, see also $[28,29]$ for a similar argumentation in isotropic elastoplasticity. In what follows, a special attention is devoted to the fibres' contribution.

Starting from the constitutive relations (18), the contribution of each family of fibres to the second Piola-Kirchhoff stress tensor is written as

$$
\mathbf{S}_{\mathrm{F}}=\mathbf{S}_{\infty_{\mathrm{F}}}+\mathbf{S}_{\mathrm{neq}_{\mathrm{F}}}=\left(\sigma_{\infty_{\mathrm{F}}}+\sigma_{\mathrm{neq}_{\mathrm{F}}}\right) \mathbf{M}_{\mathrm{F}}
$$

where we recall that the structural tensor $\mathbf{M}_{\mathrm{F}}$ is fixed. The corresponding 
(spatial) Kirchhoff stress tensor $\boldsymbol{\tau}_{\mathrm{F}}=\mathbf{F S}_{\mathrm{F}} \mathbf{F}^{T}$ is then given by

$$
\boldsymbol{\tau}_{\mathrm{F}}=\left(\sigma_{\infty_{\mathrm{F}}}+\sigma_{\mathrm{neq}_{\mathrm{F}}}\right) \mathbf{m}_{\mathrm{F}}, \quad \text { with } \quad \mathbf{m}_{\mathrm{F}}=\mathbf{F} \mathbf{M}_{\mathrm{F}} \mathbf{F}^{T} \equiv \vec{v}_{\mathrm{F}} \otimes \vec{v}_{\mathrm{F}}
$$

The material tangent modulus $\widetilde{\mathbf{C}}_{\mathrm{F}}$ is such that $\dot{\mathbf{S}}_{\mathrm{F}}=\widetilde{\mathbf{C}}_{\mathrm{F}}: \frac{1}{2} \dot{\mathbf{C}}$, while the spatial tangent modulus $\widetilde{\mathbf{C}}_{\mathrm{F}}$ is such that the Lie derivative of $\boldsymbol{\tau}_{\mathrm{F}}$ is written as $£_{\mathrm{v}} \boldsymbol{\tau}_{\mathrm{F}} \equiv \mathbf{F} \dot{\mathbf{S}}_{\mathrm{F}} \mathbf{F}^{T}=\widetilde{\mathbf{C}}_{\mathrm{F}}: \mathbf{d}$, where $\mathbf{d}$ is the spatial strain rate tensor. Recall the classical kinematical relation $\dot{\mathbf{C}}=2 \mathbf{F}^{T} \mathbf{d F}$.

Hence, ordinary time differentiation of the expression (43) leads then to the following result:

$$
\widetilde{\mathbf{C}}_{\mathrm{F}}=2 \mathbf{M}_{\mathrm{F}} \otimes\left\{\frac{\partial \sigma_{\infty_{\mathrm{F}}}}{\partial \mathbf{C}}+\frac{\partial \sigma_{\mathrm{neq}_{\mathrm{F}}}}{\partial \mathbf{C}}\right\}
$$

For the logarithmic model of section 4.1, for instance, inserting the expressions (30) and (32) into (45) gives

$$
\widetilde{\mathbf{C}}_{\mathrm{F}}=\frac{E_{\mathrm{F}}}{I_{4}} \mathbf{M}_{\mathrm{F}} \otimes \mathbf{M}_{\mathrm{F}}+E_{\mathrm{neq}_{\mathrm{F}}} \mathbf{M}_{\mathrm{F}} \otimes\left\{\frac{1}{I_{4}} \mathbf{M}_{\mathrm{F}}-\frac{2}{\lambda_{\mathrm{F}}^{\mathrm{v}}} \frac{\partial \lambda_{\mathrm{F}}^{\mathrm{v}}}{\partial \mathbf{C}}\right\}
$$

The term $\partial \lambda_{\mathrm{F}}^{\mathrm{v}} / \partial \mathbf{C}$ is computed from the algorithmic equation (40) where, again, $\sigma_{\text {neq }_{\mathrm{F}}}$ is replaced by its expression (32). The final result is given by the following simple form:

$$
\widetilde{\mathbf{C}}_{\mathrm{F}}=\left\{\frac{E_{\mathrm{F}}}{I_{4}}+\frac{E_{\mathrm{neq}_{\mathrm{F}}}}{I_{4}}-2 \alpha\right\} \mathbf{M}_{\mathrm{F}} \otimes \mathbf{M}_{\mathrm{F}}
$$

where the scalar $\alpha$ is issued from the derivation of (40), and is given for the logarithmic model by

$$
\alpha=\frac{\log \left[I_{4}\right]-2 \log \left[\lambda_{\mathrm{F}}^{\mathrm{v}}\right]+1}{\frac{2 \eta_{\mathrm{F}}}{\Delta t E_{\mathrm{neq}}}+I_{4}\left\{2+2 \log \left[\lambda_{\mathrm{F}}^{\mathrm{v}}\right]-\log \left[I_{4}\right]\right\}} .
$$

The spatial tangent modulus is also easily deduced. It remarkably has the same structure as its material counterpart: 


$$
\widetilde{\mathbf{c}}_{\mathrm{F}}=\left\{\frac{E_{\mathrm{F}}}{I_{4}}+\frac{E_{\mathrm{neq}_{\mathrm{F}}}}{I_{4}}-2 \alpha\right\} \mathbf{m}_{\mathrm{F}} \otimes \mathbf{m}_{\mathrm{F}} .
$$

For the quadratic model of section 4.2, or for the no-compression versions of both the models (section 4.3), one proceeds following similar lines starting from (45), this time by considering the expressions (34), $(36)_{1}$ and $(37)_{1}$, or $(36)_{2}$ and $(37)_{2}$.

Remark 5 In the finite element implementation, the internal variable $\mathbf{C}_{\mathrm{M}}^{\mathrm{v}}$ corresponding to the matrix and the internal variables $\lambda_{\mathrm{F}}^{\mathrm{V}}$, each one associated with one family of fibres, are stored in an element data base at the integration points level during the whole iterative process.

\section{Representative numerical examples}

The formulation proposed in this paper is illustrated below in a set of twoand three-dimensional simulations. All the model examples of section 4 have been implemented in an extended version of the CESAR-LCPC finite element software, see for example [10]. The composite materials considered herein are reinforced by one or two families of fibres along arbitrary directions. Four examples are presented. The first two are concerned with anisotropic elasticity without viscous dissipations to show, among others, the influence of the nocompression fibers' behaviour on the global response. The next two examples show the main viscous characteristics of the present formulation.

\subsection{Fibre directions and no-compression behaviour influences}

In this first example, the peculiar anisotropic behaviour is shown for pure elasticity, i.e. at thermodynamic equilibrium under slow loading velocities. Hence viscous effects are neglected for the moment. The influence of the fibre direction on the overall behaviour is discussed together with the fact that whether or not the no-compression behaviour is taken into account.

We consider the $(100 \times 25) \mathrm{mm}^{2}$ rectangular specimen of figure 1 . The boundary conditions are such that the right edge is loaded by imposing the horizontal displacement $\bar{u}_{\text {imp }}$. The specimen is discretized with $40 \times 10$ classical 4-nodes isoparametric elements using the linear interpolation. The middle node of the left edge is fixed in the vertical direction to avoid any rigid body motion and a plane strain condition is adopted. The material is reinforced by only one family of fibres characterized by the angle $\theta$ with the horizontal direction (see figure 1). 
Figure 1. Geometry and boundary conditions. Fibre-reinforced material with one family of fibres.

For the matrix material, we use the following bulk and shear moduli (see (26)) : $\kappa_{\mathrm{M}}=1400 \mathrm{MPa}$ and $\mu_{\mathrm{M}}=3.2 \mathrm{MPa}$, hence a quasi-incompressible behaviour is chosen (see remark 2), and for the Ogden's parameters we use:

$$
\begin{aligned}
& c_{1}=1.9384, \quad c_{2}=.014, \quad c_{3}=.0474, \\
& m_{1}=1.30, \quad m_{2}=5.00, \quad m_{3}=-2.00 \text {. }
\end{aligned}
$$

For the fibres, we adopt in this example the logarithmic model of section 4.1. The Young's modulus is choosen as $E_{\mathrm{F}}=35 \mathrm{MPa}$.

Figure 2 shows the results of a first series of displacement driven computations together with the deformed configurations at the ultimate loads. That is, the resultant reaction force-displacement curves until the imposed displacements $\bar{u}_{\text {imp }}=60 \mathrm{~mm}$ (stretching up to $\approx 60 \%$ ), and for different orientations $\theta$ of the fibres without taking into account the no-compression behaviour. The loading history consists of 30 steps of equal displacement increments $\Delta \bar{u}_{\text {imp }}=2 \mathrm{~mm}$.

Figure 2. Resultant load-displacement curves for different orientations $\theta$ of the fibres without no-compression effect.

These results show the strong influence of the fibres' directions on the global response of the streep. In fact, the right edge can move downwards (for $\theta=10^{\circ}$ or $30^{\circ}$ for instance), or upwards (for $\theta=80^{\circ}$ like the figure shows). And for $\theta=0^{\circ}$ and $90^{\circ}$ we obtain logically uniform deformations. Moreover, for $\theta$ greater than $45^{\circ}$ until $90^{\circ}$, the initial stiffness of the specimen increases. This is because, with the model employed herein, the fibres can carry loads in compression as the sample is submitted to striction. Similar results have been obtained in elastoplasticity, see [11].

However, in practice, the fibres cannot carry loads in compression. Figure 3 shows then the results of the same computations with the same material parameters, this time with the no-compression behaviour for the fibres as described in section 4.3. We obtain the same results as for the precedent computations for orientations $\theta$ under $45^{\circ}$ (in fact, the fibres are submitted to traction in this case). And for $\theta$ greater than $45^{\circ}$, only the matrix contributes to the mechanical response of the specimen as the fibres are contracted. Moreover for this latter case, the corresponding deformed configurations are all uniform $\left(\theta=60^{\circ}, 80^{\circ}\right.$ and $90^{\circ}$ in figure 3 for instance). 
Figure 3. Resultant load-displacement curves for different orientations $\theta$ of the fibres with the no-compression effect.

In all the next examples, we use only the no-compression versions of the constitutive models for the fibres.

\subsection{Inflation of a fibre-reinforced rubber tube}

We consider in this example the elastic response of a long circular tube continuously reinforced by two families of fibres. The fibres are symmetrically arranged with respect to the axial direction in a helical manner (see figure 4). In the reference configuration, the internal radius is $R_{i}=100 \mathrm{~mm}$ and the thickness is $e=5 \mathrm{~mm}$. The length of the portion we consider in the following computations is initially $H=200 \mathrm{~mm}$. Almost the same problem has been considered in [8]. Structures of this type are frequently employed in industrial applications. Moreover, in biomechanics, many living organisms are cylinders reinforced by collagen fibres, like the blood vessels for example.

Figure 4. Fibre-reinforced tube. Geometry, finite element discretization and loading conditions.

One quarter of the tube is discretized with $10 \times 20$ hexahedral 8-nodes trilinear isoparametric elements and two elements through the thickness, i.e. a total of 400 elements (see figure 4). The sample is subjected to an increasing internal pressure $p$ and a uniform axial force, due to the internal pressure, with resultant $F \equiv F\left(p, r_{i}\right)=p \pi r_{i}^{2}$. Here $r_{i}$ is the internal radius in the deformed configuration. For the matrix, use is made of the material parameters of the precedent example $\left(\kappa_{\mathrm{M}}=1400 \mathrm{MPa}, \mu_{\mathrm{M}}=3.2 \mathrm{MPa}\right.$ and the Ogden's parameters (50)). The two families of fibres have the same behaviours. We use this time the no-compression version of the quadratic model of section 4.2 with a Young's modulus $E_{\mathrm{F}}=250 \mathrm{MPa}$.

Figure 5 shows the evolutions of circumferential stretch $\lambda_{\theta}$ when the internal pressure increases until $p=100$ bar for different orientations of the fibres' angle $\gamma$ with respect to the circumferential direction (see figure 4). The loading history consists of 100 steps of equal internal pressure increments $\Delta p=1$ bar. And figure 6 shows the evolutions of the corresponding longitudinal stretch $\lambda_{z}$. Here $\lambda_{\theta}$ is given as

$$
\lambda_{\theta}=\frac{r}{R}=1+\frac{u_{r}}{R}
$$


where $r$ is the actual mean radius of the tube, and $R=R_{i}+e / 2$ is the initial mean radius. $R=102.5 \mathrm{~mm}$ in our example.

Figure 5. Inflation of a fibre-reinforced tube. Circumferential stretch $\lambda_{\theta}$ for different orientation $30^{\circ} \leq \gamma \leq 40^{\circ}$.

Figure 6. Inflation of a fibre-reinforced tube. Longitudinal stretch $\lambda_{z}$ for different orientation $30^{\circ} \leq \gamma \leq 40^{\circ}$.

As for example in [8], we observe here again the typical stretch inversion phenomenon in the low pressure domain $(0 \leq p \leq 20$ bar in our example). Following the computed results, the diameter of the tube starts decreasing $\left(\lambda_{\theta}<1\right)$ while the longitudinal stretch $\lambda_{z}$ increases for fibre orientations $\gamma$ around $30^{\circ}$. Conversely, for orientations around $40^{\circ}$, the length of the tube starts decreasing $\left(\lambda_{z}<1\right)$ while the diameter of the tube increases.

\subsection{Relaxation tests of a rectangular reinforced rubber streep}

To show in a simple manner the main viscous characteristics of the present formulation, we consider again the rectangular rubber strip of the first example with the same boundary conditions and finite element discretization. We include this time the viscous phenomena in the fibre-reinforced constitutive relations. For the equilibrium part, the same material parameters are used as in section 6.1. That is: $\kappa_{\mathrm{M}}=1400 \mathrm{MPa}, \mu_{\mathrm{M}}=3.2 \mathrm{MPa}$ and the Ogden's parameters given by (50) for the matrix, and $E_{\mathrm{F}}=35 \mathrm{MPa}$ for the single fibre family (the no-compression logarithmic model is employed for the fibres).

The following material parameters associated with the non-equilibrium part of the model are used: for the matrix (see (27) and (28)), $\kappa_{\mathrm{neq}_{\mathrm{M}}}=1050 \mathrm{MPa}$, $\mu_{\text {neq }_{\mathrm{M}}}=2.4 M P a, \eta_{\mathrm{v}_{\mathrm{M}}}=21.10^{3} \mathrm{MPa} s$ and $\eta_{\mathrm{d}_{\mathrm{M}}}=48 \mathrm{MPa} s$, and for the fibres, $E_{\text {neq }_{\mathrm{F}}}=24 \mathrm{MPa}$ and $\eta_{\mathrm{F}}=480 \mathrm{MPa} s$ (see (37) ${ }_{1}$ and (25)). Observe that, in this example, these material data are such that the relaxation times of the volumetric and deviatoric matrix contributions, and of the fibre component are the same : $\tau=\eta_{\mathrm{v}_{\mathrm{M}}} / \kappa_{\text {neq }_{\mathrm{M}}}=\eta_{\mathrm{d}_{\mathrm{M}}} / \mu_{\mathrm{neq}_{\mathrm{M}}}=\eta_{\mathrm{F}} / E_{\text {neq }_{\mathrm{F}}}=20 \mathrm{~s}$. However, this does not mean that the relaxation time of the whole composite is $20 \mathrm{~s}$ as the following numerical results show.

Figure 7 illustrates the results of displacement driven computations at different velocities, for different fibre orientations $\theta$ (see figure 1), and with a period of relaxation of $30 \mathrm{~s}$ at the end of each loading history. The loadings consist of 30 equal displacement increments $\Delta \bar{u}_{\text {imp }}=2 \mathrm{~mm}$ with two different time steps: $\Delta t=1 \mathrm{~s}\left(\right.$ for $\left.\dot{\bar{u}}_{\text {imp }}=2 \mathrm{~mm} / \mathrm{s}\right)$, and $\Delta t=0.4 \mathrm{~s}$ for $\left(\dot{\bar{u}}_{\mathrm{imp}}=5 \mathrm{~mm} / \mathrm{s}\right)$. 
Figure 7. Resultant force-imposed displacement curves at different loading velocities $\dot{\bar{u}}_{\text {imp }}$ and for different orientations $\theta$ of the fibres.

We find the typical responses of viscous materials. That is, the load carrying capacity of the streep increases with inceasing loading velocity. Moreover, for orientations of the fibres $\theta$ greater that $45^{\circ}$ (for instance $\theta=80^{\circ}$ in figure 7 ), the response is the one due to the isotropic matrix compound only as the fibres are submitted to compression in this case.

Finally, figure 8 illustrates the corresponding evolutions of the reaction force during the period of relaxation at the end of each loading history, i.e. at $\bar{u}_{\text {imp }}=60 \mathrm{~mm}$. They are compared to their respective asymptotic values at thermodynamic equilibrium (see figure 3 for $\theta=10^{\circ}$ and $80^{\circ}$ ). In figure 8 , the initial time $t=0$ corresponds to the moment where the $30 \mathrm{~s}$ relaxation period starts.

Figure 8. Relaxation at $\bar{u}_{\text {imp }}=60 \mathrm{~mm}$ for different velocities and fibre orientations $\theta$.

\subsection{Cyclic inflation of a fibre-reinforced rubber tube}

In this last example, we consider viscous effects in the fibre-reinforced rubber tube of section 6.2 with the same finite element discretization, i.e. one quarter of the specimen is considered with $10 \times 20 \times 2$ hexahedral elements. For the equilibrium part, the same parameters are used as in the purely elastic case: $\kappa_{\mathrm{M}}=1400 \mathrm{MPa}, \mu_{\mathrm{M}}=3.2 \mathrm{MPa}$ and the Ogden's parameters given by(50) for the matrix, and $E_{\mathrm{F}}=250 M P a$ for the two families of fibres.

Associated with the non-equilibrium part, the following material parameters are used: $\kappa_{\text {neq }_{\mathrm{M}}}=1662.5 \mathrm{MPa}, \mu_{\text {neq }_{\mathrm{M}}}=3.8 \mathrm{MPa}, \eta_{\mathrm{v}_{\mathrm{M}}}=33.2510^{3} \mathrm{MPa} \mathrm{s}$ and $\eta_{\mathrm{d}_{\mathrm{M}}}=76 \mathrm{MPa} s$ for the matrix, and $E_{\mathrm{neq}_{\mathrm{F}}}=300 \mathrm{MPa}$ and $\eta_{\mathrm{F}}=6000 \mathrm{MPas}$ for each fibres' family.

The tube is submitted to an internal pressure $p$ and a uniform axial force (due to the internal pressure) of resultant $F=p \pi r_{i}^{2}$ (see figure 4). At time $t=0$, the pressure is first increased with a constant velocity $\dot{p}=1 \mathrm{bar} / \mathrm{s}$ until it reaches the value $p=70 \mathrm{bar}$. This is achieved with 70 increments $\Delta p=1 \mathrm{bar}$ and equal time steps $\Delta t=1 \mathrm{~s}$. Then, a cyclic loading is applied. The pressure $p$ follows a linear increasing/decreasing function with an amplitude of $30 \mathrm{bar}$ and a period $T=15 \mathrm{~s}$, i.e. increasing and decreasing pressure between 40 and $100 \mathrm{bar}$ with velocities of $8 \mathrm{bar} / \mathrm{s}$ (see figure 9 ). When computing this cyclic loading, use has been made of pressure increments $|\Delta p|=2$ bar with equal time steps $\Delta t=0.25 \mathrm{~s}$. 
Under this loading history, figure 9 shows the evolutions of the circumferential stretch $\lambda_{\theta}$ versus the internal pressure $p$ for two different orientations of the fibres with respect to the circumferential direction: $\gamma=30^{\circ}$ and $\gamma=40^{\circ}$ (see figure 4 ). Here also we observe the stretch inversion phenomenon in the low pressure domain.

Figure 9. Cyclic inflation of a viscous fibre-reinforced tube. Loading history and circumferential stretch $\lambda_{\theta}$ for $\gamma=30^{\circ}$ and $40^{\circ}$.

For instance, figure 10 illustrates the evolution of $\lambda_{\theta}$ for $\gamma=30^{\circ}$ versus the relative time $t_{0}$ with respect to moment at which the cyclic loading starts. In our example, we have $t_{0}=t-70 \mathrm{~s}$. The circumferential stretch $\lambda_{\theta}$ is 1.075 at $t_{0}=0$ for $p=70$ bar, and it varies between 1.064 and 1.107 during the first cycle. After 6 time periods, its value is 1.095 for $p=70$ bar at it varies between 1.075 and 1.122 .

Figure 10. Cyclic evolution of the circumferential stretche $\lambda_{\theta}$ versus the relative time $t_{0}$ for $\gamma=30^{\circ}$.

\section{Conclusions}

In this paper a viscoelastic formulation for fibre-reinforced materials that experience finite strains has been presented. These composites are thought to be the superposition of an isotropic matrix and further sets of fibres. Each family of fibres is considered as a one-dimensional continuum at the macroscopic level, and the anisotropy of the whole composite is induced by their directional responses.

The viscoelastic kinematics adopted here is based on an extension of the hypothesis of the multiplicative decomposition of the deformation gradient into an elastically relaxing part and a viscous part. This nowadays well known assumption has up to now been applied only for the isotropic case. The different components of the fibre-matrix material are then implicitly linked by the fact that this kinematics apply to all of them. The constitutive relations and the correct form of the governing equations are established on the basis of the continuum thermodynamics. In particular for the fibres, the viscous evolutions reduce to single scalar equations, one for each fibres' family. Some model examples have been presented among many other possible choices. Again for the fibres, as in practice they cannot carry loads in compression, no-compression models has been proposed and demonstrated numerically. 
From the numerical point of view within the finite element method, the effort is of the order of that of isotropic viscoelastic computations. The additional fibre contributions reduce to the resolution of discrete single-scalar equations, one for each set of fibres, together with the corresponding contributions to the algorithmic tangent moduli. Numerical examples have shown the efficiency of the whole numerical procedure where anisotropic elasticity is included as a special case.

\section{References}

[1] J. Bonet and A.J. Burton. A simple orthotropic, transversely isotropic hyperelastic constitutive equation for large strain computations. Computer Methods in Applied Mechanics and Engineering, 162:151-164, 1998.

[2] B.D. Coleman and M.E. Gurtin. Thermodynamics with internal variables. Journal of Chemics and Physics, 47:597-613, 1967.

[3] B.D. Coleman and W. Noll. Foundation of linear viscoelasticity. Reviews of Modern Physics, 33:239-249, 1961.

[4] Y.C. Fung. A first course in continuum mechanics for physical and biological engineers and scientists. Prentice-Hall, Englewood-Cliffs, 1994.

[5] S. Govindjee and S. Reese. A presentation and comparison of two large deformation viscoelastic models. ASME Journal of Engineering Materials and Technology, 119:251-255, 1997.

[6] C.T. Herakovich. Mechanics of fibrous composites. John Wiley and Sons Inc., New York, 1998.

[7] G.A. Holzapfel. Nonlinear solid mechanics. A continuum appraoch for engineering. John Wiley and Sons, Ltd, Chichester, West Sussex, UK, 2000.

[8] G.A. Holzapfel and T.C. Gasser. A viscoelastic model for fibre-reinforced composites at finite strains: Continuum basis, computational aspects and applications. Computer Methods in Applied Mechanics and Engineering, 190:4379-4403, 2001.

[9] G.A. Holzapfel, T.C. Gasser, and M. Stadler. A structural model for the viscoelastic behavior of arterial walls: Continuum formulation and finite element analysis. European Journal of Mechanics A/Solids, 21:441-463, 2002.

[10] P. Humbert, A. Dubouchet, G. Fezans, and D. Remaud. CESAR-LCPC, un progiciel de calcul dédié au génie civil. Bulletin des Laboratoires des Ponts et Chaussées, 256-257:7-37, 2005.

[11] S. Klinkel, C. Sansour, and W. Wagner. An anisotropic fibre-matrix material model at finite elastic-plastic strains. Computational Mechanics, 35:409-417, 2005. 
[12] P. LeTallec, C. Rahier, and A. Kaiss. Three-dimensional incompressible viscoelasticity in large strains: formulation and numerical approximation. Computer Methods in Applied Mechanics and Engineering, 109:223-258, 1993.

[13] A. Lion. A constitutive model for carbon black filled rubber. experimental investigations and mathematical representations. Continuum Mechanics and Thermodynamics, 8:153-169, 1996.

[14] J. Lubliner. A model of rubber viscoelasticity. Mechanics Research Communications, 12:93-99, 1985.

[15] G.A. Maugin. Eshelby stress in elastoplasticity and ductile fracture. International Journal of Plasticity, 10:393-408, 1994.

[16] C. Miehe. Aspects of the formulation and finite element implementation of large strain isotropic elasticity. International Journal of Numerical Methods in Engineering, 37:1981-2004, 1994.

[17] B. Nedjar. Frameworks for finite strain viscoelastic-plasticity based on multiplicative decompositions. Part I: Continuum formulations. Computer Methods in Applied Mechanics and Engineering, 191:1541-1562, 2002.

[18] B. Nedjar. Frameworks for finite strain viscoelastic-plasticity based on multiplicative decompositions. Part II: Computational aspects. Computer Methods in Applied Mechanics and Engineering, 191:1563-1593, 2002.

[19] R.W. Ogden. Nonlinear elastic deformations. Ellis Horwood Ltd., West Susex, England, 1984.

[20] S. Reese. Meso-macro modelling of fibre-reinforced rubber-like composites exhibiting large elastoplastic deformation. International Journal of Solids and Structures, 40:951-980, 2003.

[21] S. Reese and S. Govindjee. A theory of finite viscoelasticity and numerical aspects. International Journal of Solids and Structures, 35(26-27):3455-3482, 1998.

[22] S. Reese, T. Raible, and P. Wriggers. Finite element modelling of orthotropic material behaviour in pneumatic membranes. International Journal of Solids and Structures, 38:9525-9544, 2001.

[23] C. Sansour and J. Bocko. On the numerical implications of multiplicative inelasticity with an anisotropic elastic constitutive law. International Journal for Numerical Methods in Engineering, 58:2131-2160, 2003.

[24] C. Sansour, I. Karsaj, and J. Soric. A formulation of anisotropic continuum elastoplasticity at finite strains. Part I: Modelling. International Journal of Plasticity, 22:2346-2365, 2006.

[25] C. Sansour and G. Kollmann. Large viscoplastic deformations of shells. theory and finite element formulation. Computational Mechanics, 21:512-525, 1998. 
[26] J. Sidoroff. Un modèle viscoélastique nonlinéaire avec configuration intermédiaire. Journal de Mécanique, 13:679-713, 1974.

[27] J.C. Simo. On a fully three-dimensional finite-strain viscoelastic damage model: formulation and computational aspects. Computer Methods in Applied Mechanics and Engineering, 60:153-173, 1987.

[28] J.C. Simo. Algorithms for static and dynamic multiplicative plasticity that preserve the classical return mapping schemes of the infinitesimal theory. Computer Methods in Applied Mechanics and Engineering, 99:61-112, 1992.

[29] J.C. Simo. Numerical analysis and simulation of plasticity. In P.G. Ciarlet and J.L. Lions, editors, Handbook of Numerical Analysis, vol. VI, pages 183-499. North-Holland, 1998.

[30] J.C. Simo and R.L. Taylor. Quasi-incompressible finite elasticity in principal stretches. Continuum basis and numerical algorithms. Computer Methods in Applied Mechanics and Engineering, 85:273-310, 1991.

[31] A.J.M. Spencer. Constitutive theory for strongly anisotropic solids. In A.J.M. Spencer, editor, Continuum theory of the mechanics of fibre-reinforced composites, CISM cources and lectures No. 282. Springer, Wien, 1984.

[32] A.J.M. Spencer. A theory of viscoplasticity for fabric-reinforced composites. Journal of the Mechanics and Physics of Solids, 49:2667-2687, 2001.

[33] C. Truesdell and W. Noll. The nonlinear field theories of mechanics. In S. Fluegge, editor, Handbuch der Physik Bd. III/3. Springer-Verlag, Berlin, 1965.

[34] J.A. Weiss, B.N. Maker, and S. Govindjee. Finite element implementation of incompressible, transversely isotropic hyperelasticity. Computer Methods in Applied Mechanics and Engineering, 135:107-128, 1996. 


\section{Figure captions}

Fig. 1: Geometry and boundary conditions. Fibre-reinforced material with one family of fibres.

Fig. 2: Resultant load-displacement curves for different orientations $\theta$ of the fibres without no-compression effect.

Fig. 3: Resultant load-displacement curves for different orientations $\theta$ of the fibres with the no-compression effect.

Fig. 4: Fibre-reinforced tube. Geometry, finite element discretization and loading conditions.

Fig. 5: Inflation of a fibre-reinforced tube. Circumferential stretch $\lambda_{\theta}$ for different orientations $30^{\circ} \leq \gamma \leq 40^{\circ}$.

Fig. 6: Inflation of a fibre-reinforced tube. Longitudinal stretch $\lambda_{z}$ for different orientations $30^{\circ} \leq \gamma \leq 40^{\circ}$.

Fig. 7: Resultant force-imposed displacement curves at different loading velocities and for different orientations $\theta$ of the fibres.

Fig. 8: Relaxation at $\bar{u}_{\text {imp }}=60 \mathrm{~mm}$ for different velocities and fibre orientations $\theta$.

Fig. 9: Cyclic inflation of a viscous fibre-reinforced tube. Loading history and circumferential stretch $\lambda_{\theta}$ for $\gamma=30^{\circ}$ and $40^{\circ}$.

Fig. 10: Cyclic evolution of the circumferential stretch $\lambda_{\theta}$ versus the relative time $t_{0}$ for $\gamma=30^{\circ}$. 


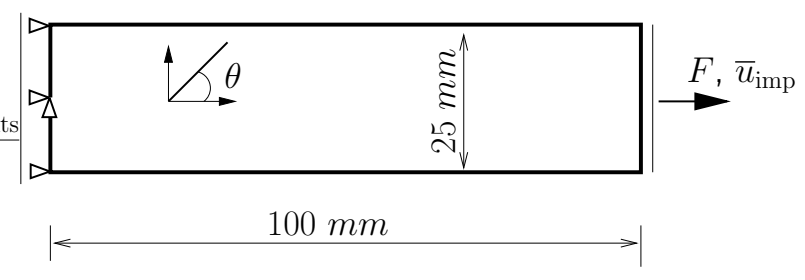

Fig. 1. 


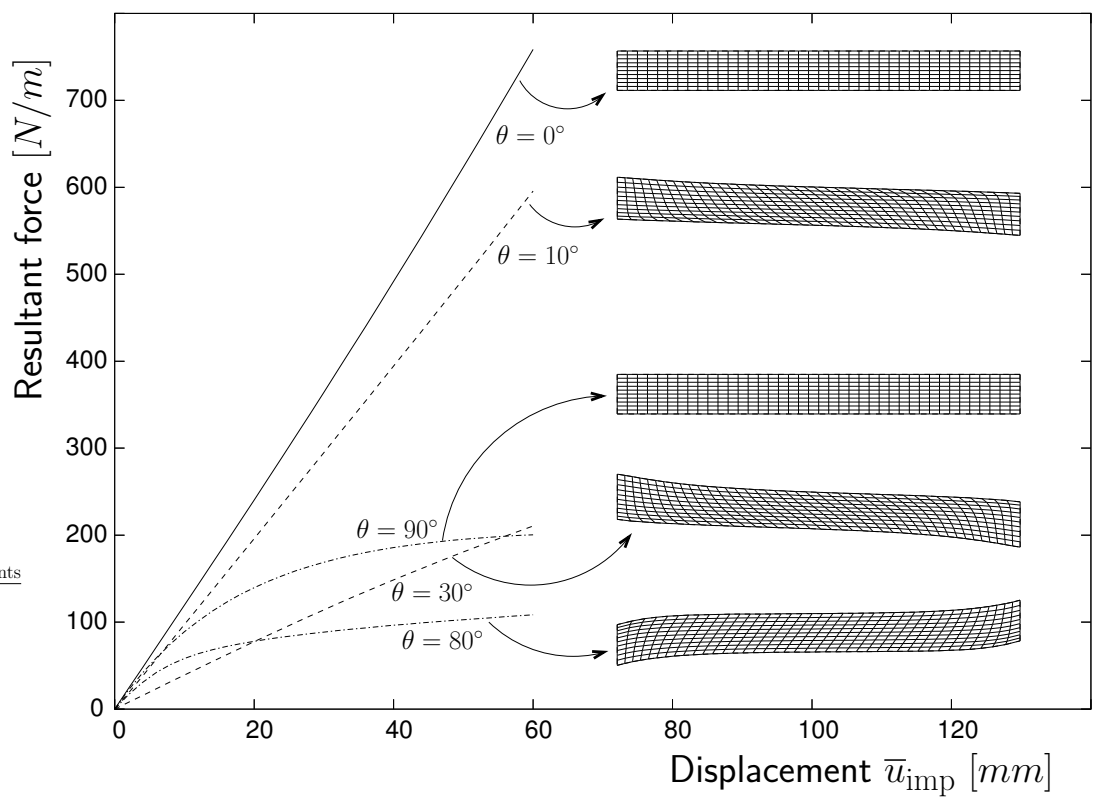

Fig. 2. 


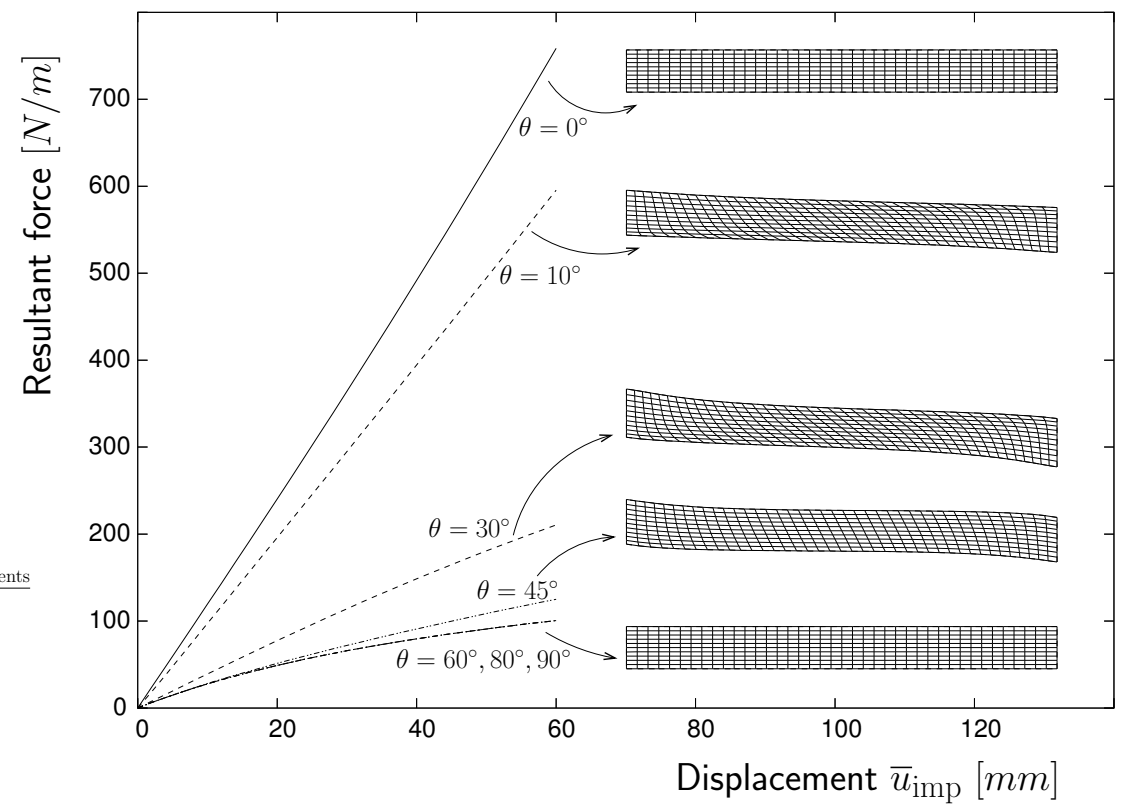

Fig. 3. 

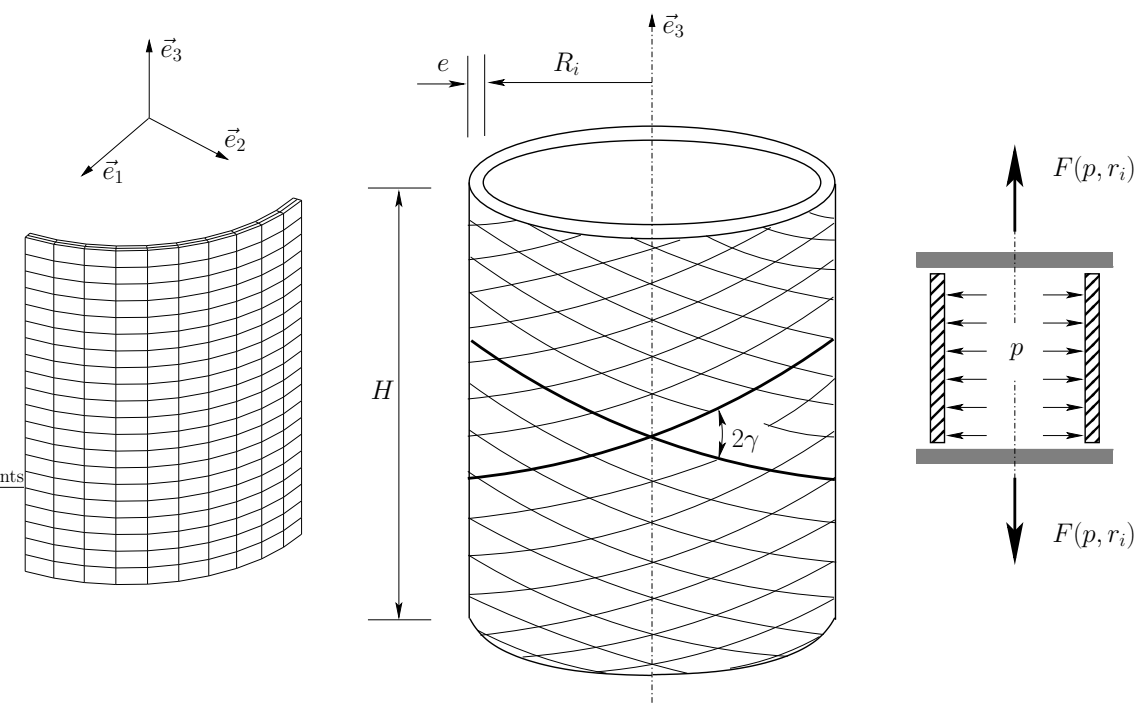

Fig. 4. 


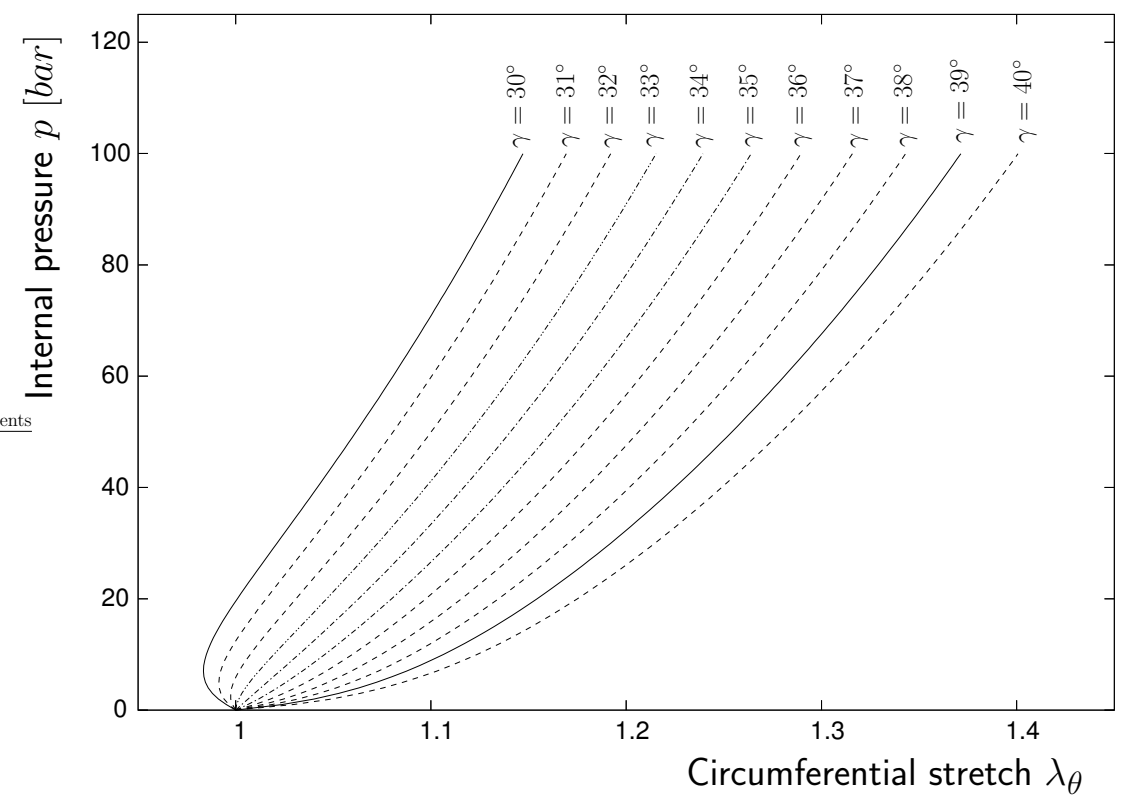

Fig. 5. 


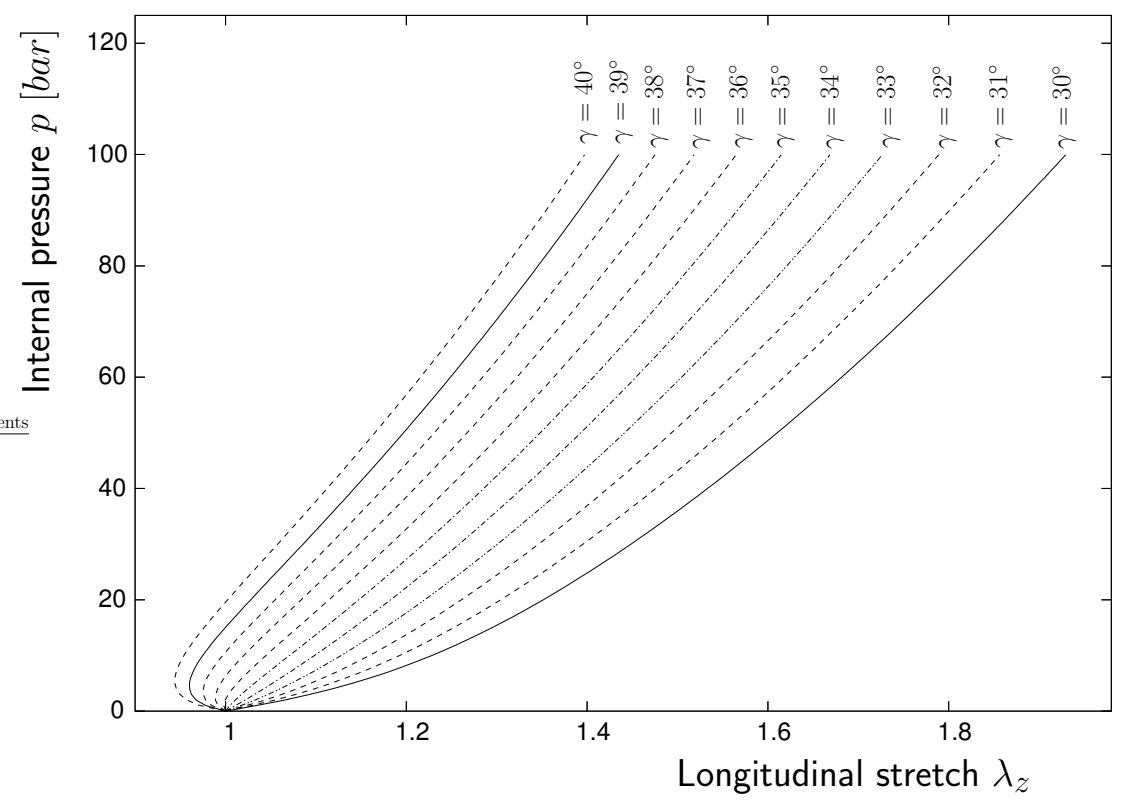

Fig. 6 . 


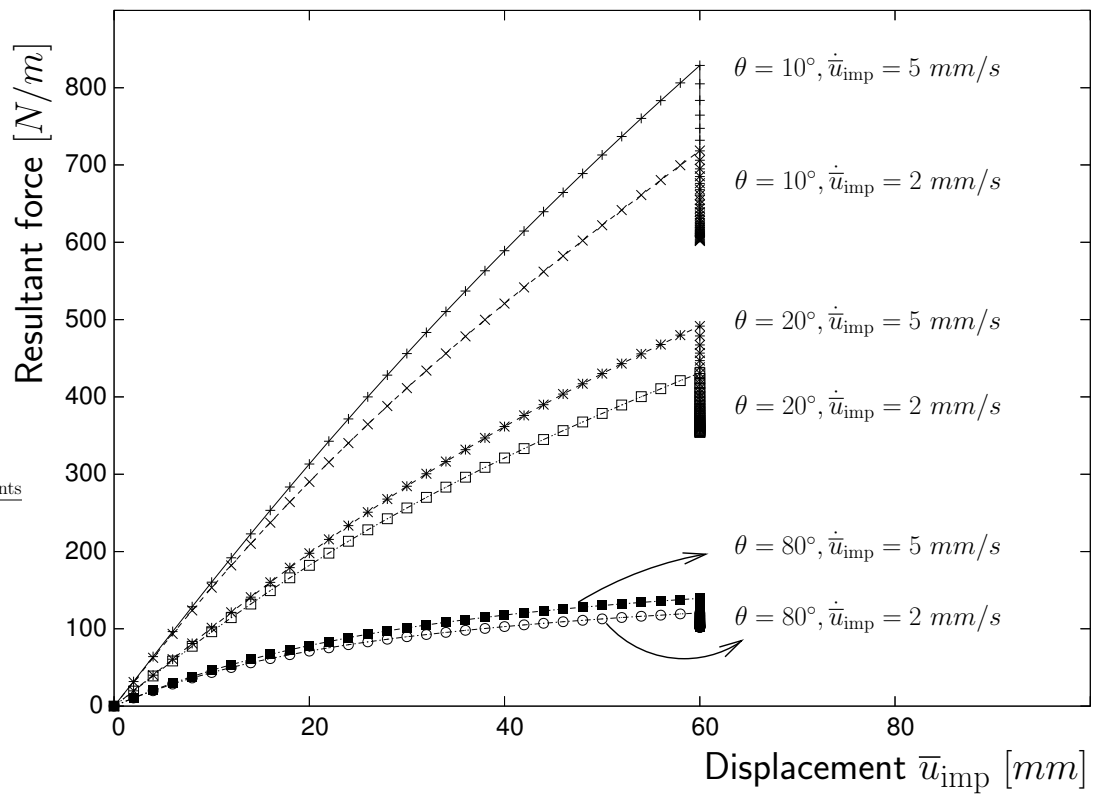

Fig. 7. 


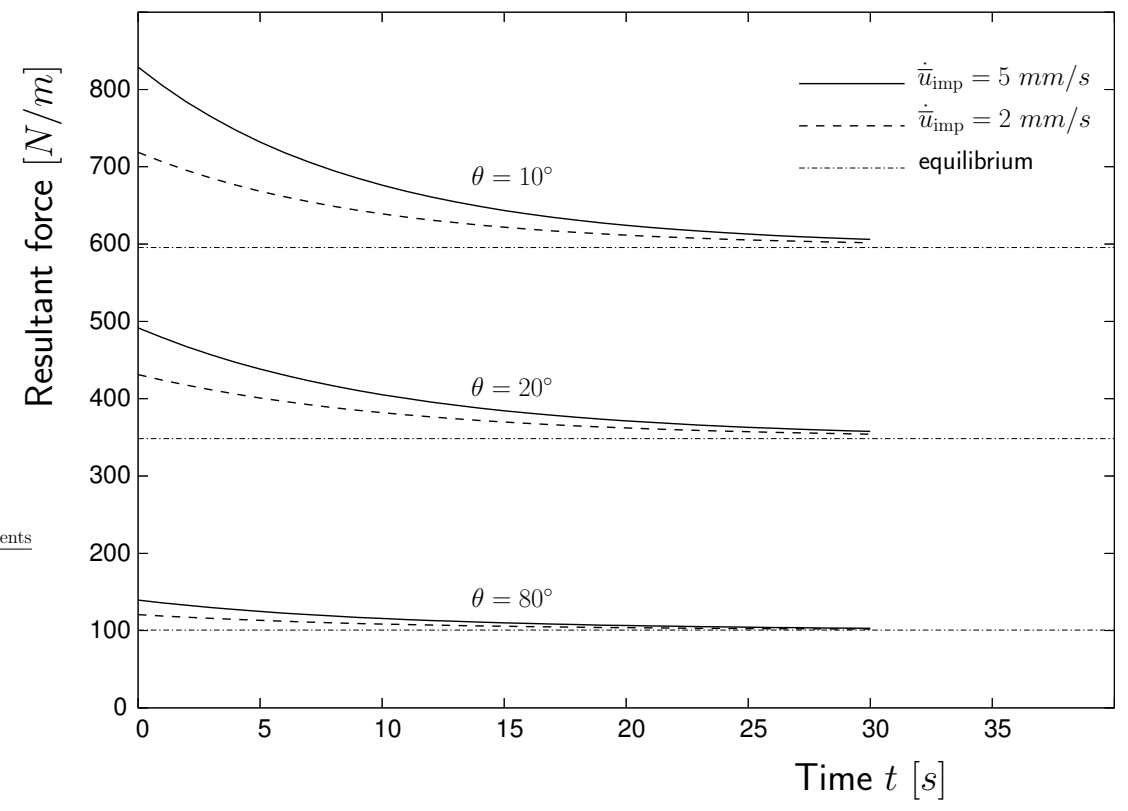

Fig. 8 . 


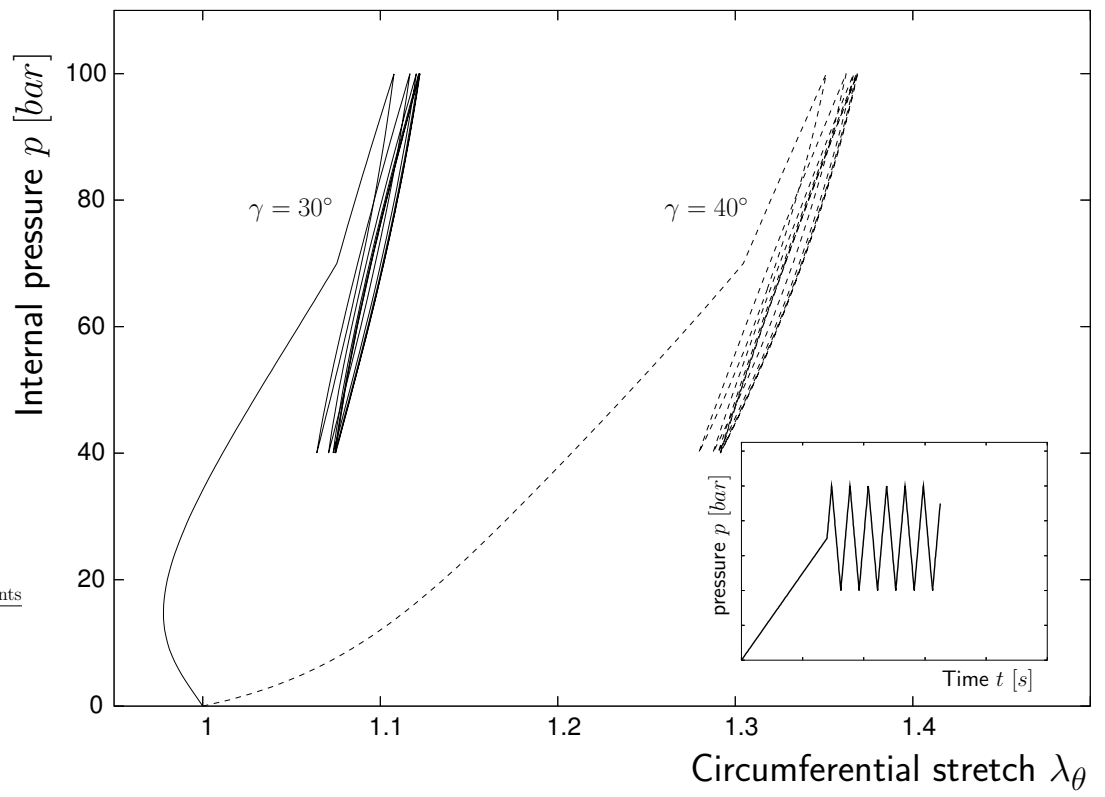

Fig. 9. 


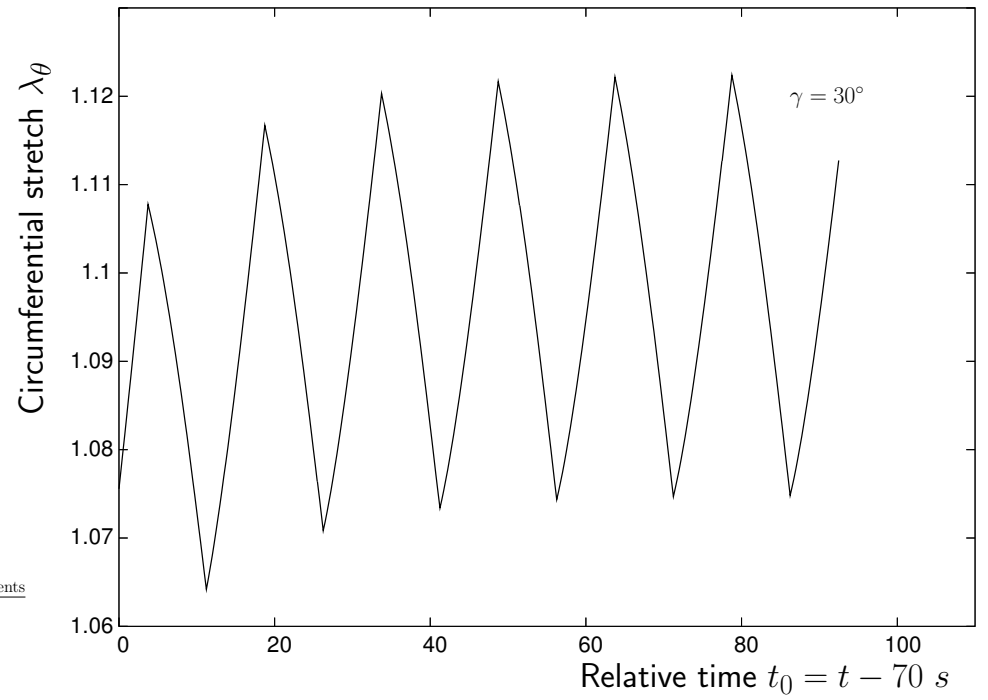

Fig. 10. 